CRYSTALLOGRAPHIC COMMUNICATIONS

ISSN 2056-9890

Received 13 July 2020

Accepted 4 August 2020

Edited by G. Diaz de Delgado, Universidad de Los Andes, Venezuela

Keywords: crystal structure; manganese(I) complex; bipyridyl ligand; biquinolyl ligand; ester substituent.

CCDC references: 2021226; 2021225

Supporting information: this article has supporting information at journals.iucr.org/e

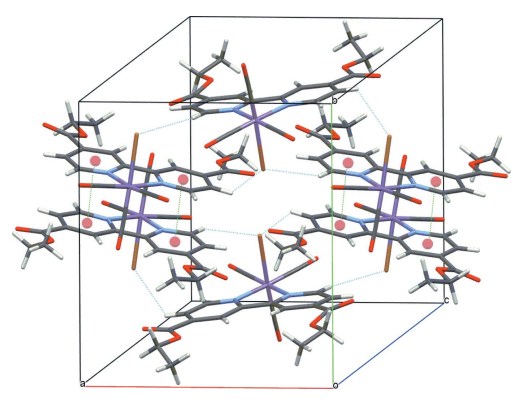

OPEN $\odot$ ACCESS

\section{Synthesis and crystal structures of manganese(I) carbonyl complexes bearing ester-substituted $a$-diimine ligands}

\author{
Takatoshi Kanno, $^{a}$ Tsugiko Takase $^{\mathrm{b}}$ and Dai Oyama ${ }^{\mathrm{b} *}$
}

${ }^{a}$ Graduate School of Science and Engineering, Fukushima University, 1 Kanayagawa, Fukushima 960-1296, Japan, and ${ }^{\mathbf{b}}$ Department of Natural Sciences and Informatics, Fukushima University, 1, Kanayagawa, Fukushima 960-1296, Japan. *Correspondence e-mail: daio@sss.fukushima-u.ac.jp

The crystal structures of two manganese(I) complexes with ester-substituted bipyridine or biquinoline supporting ligands are reported, namely, facbromidotricarbonyl(diethyl 2,2'-bipyridine-4,4'-dicarboxylate- $\kappa^{2} N, N^{\prime}$ )manganese(I), $\left[\mathrm{MnBr}\left(\mathrm{C}_{16} \mathrm{H}_{16} \mathrm{~N}_{2} \mathrm{O}_{4}\right)(\mathrm{CO})_{3}\right], \mathbf{I}$, and fac-bromidotricarbonyl(diethyl 2,2'-biquinoline-4,4'-dicarboxylate- $\left.\kappa^{2} N, N^{\prime}\right)$ manganese(I), $\left[\mathrm{MnBr}\left(\mathrm{C}_{24} \mathrm{H}_{20} \mathrm{~N}_{2} \mathrm{O}_{4}\right)\right.$ $\left.(\mathrm{CO})_{3}\right]$, II. In both complexes, the manganese(I) atom adopts a distorted octahedral coordination sphere defined by three carbonyl $\mathrm{C}$ atoms, $\mathrm{a} \mathrm{Br}^{-}$anion and two $\mathrm{N}$ atoms from the chelating $\alpha$-diimine ligand. Both complexes show fac configurations of the carbonyl ligands. In I, the complex molecules are linked by $\mathrm{C}-\mathrm{H} \cdots \mathrm{Br}$ hydrogen bonds and aromatic $\pi-\pi$ contacts. In II, intramolecular $\mathrm{C}-\mathrm{H} \cdots \mathrm{O}$ hydrogen bonds are present as well as intermolecular $\mathrm{C}-\mathrm{H} \cdots \mathrm{O}$ and $\mathrm{C}-\mathrm{H} \cdots \mathrm{Br}$ hydrogen bonds and $\pi-\pi$ interactions.

\section{Chemical context}

Similar to carbonyl complexes of precious metals, such as ruthenium and rhenium, those with less expensive manganese are attracting attention for their application in $\mathrm{CO}_{2}$ reduction catalysts (Bourrez et al., 2011) and as CO-releasing molecules (CORMs) under external stimuli (Chakraborty et al., 2014a). For example, CORMs using manganese(I) carbonyl complexes controllably release $\mathrm{CO}$ by photoirradiation (Motterlini et al., 2002). Considering their application in vivo, photo-CORMs are expected to utilize light at lower energy. In general, extended $\pi$-conjugation systems in organic ligands lead to redshifts of charge-transfer (CT) transition bands of manganese(I) carbonyl complexes (Chakraborty et al., 2014b). Therefore, it is essential to investigate the relationship between molecular structures including $\pi$-conjugation systems and photophysical properties.

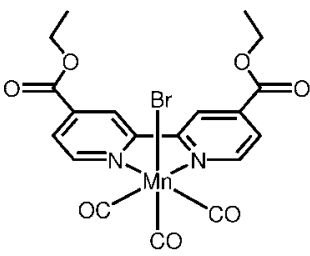

I

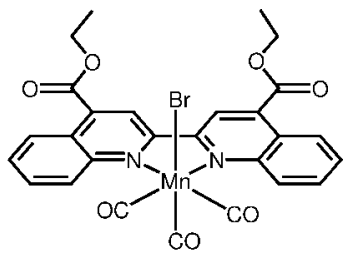

II
Thus, we focused on the comparison of bipyridines, which are prototypes of the $\alpha$-diimine ligand, and biquinolines with a more extended $\pi$-conjugation system. In addition, the introduction of ester groups into these ligands allows chemical 
adsorption with various metal oxides (Ardo \& Meyer, 2009; Zhang et al., 2006). In this study, we synthesized manganese(I) tricarbonyl complexes bearing two types of $\alpha$-diimine compounds, which contain both an ester substituent and different $\pi$-conjugation systems, viz. diethyl 2,2'-bipyridine4,4'-dicarboxylate (debpy) and diethyl 2,2'-biquinoline-4,4'didicarboxylate (debqn): $f a c$ - $\left[\operatorname{MnBr}(\mathrm{CO})_{3}(\right.$ debpy) $]$ (I) and $f a c-\left[\operatorname{MnBr}(\mathrm{CO})_{3}(\right.$ debqn $\left.)\right]($ II $)$. We successfully compared their crystal structures and photophysical properties. As expected, a CT band shift in the visible region was confirmed, depending on the size of the $\pi$-conjugation system in $\alpha$-diimine ligands. This finding will provide information in the future design of suitable complexes for a variety of photoreactions (Chakraborty et al., 2014b).

\section{Structural commentary}

The molecular structures of compounds I and II are shown in Figs. 1 and 2, respectively. In both complexes, the manganese(I) atoms exhibit distorted octahedral coordination geometries and display primary coordination spheres that are similar to those reported for other structurally related complexes (Chakraborty et al., 2014a; Walsh et al., 2015). The metal-ligand bond lengths are similar to those previously reported for compounds of this type; in $\mathbf{I}$, the $\mathrm{Mn}-\mathrm{N}$ bond lengths are 2.046 (3) and 2.047 (2) $\AA$, while in II, the $\mathrm{Mn}-\mathrm{N}$ bond lengths are 2.063 (2) and 2.068 (2) $\AA$. In I and II, the $f a c$ configuration of three $\mathrm{CO}$ ligands around the central manganese(I) atom is in agreement with their IR data. On the basis of their bond parameters, all CO ligands have typical triplebond characters.

The torsion angles between the equatorial plane and the debpy pyridyl ring in $\mathbf{I}(\mathrm{C} 3-\mathrm{Mn} 1-\mathrm{N} 1-\mathrm{C} 8$ and $\mathrm{C} 2-\mathrm{Mn} 1-$

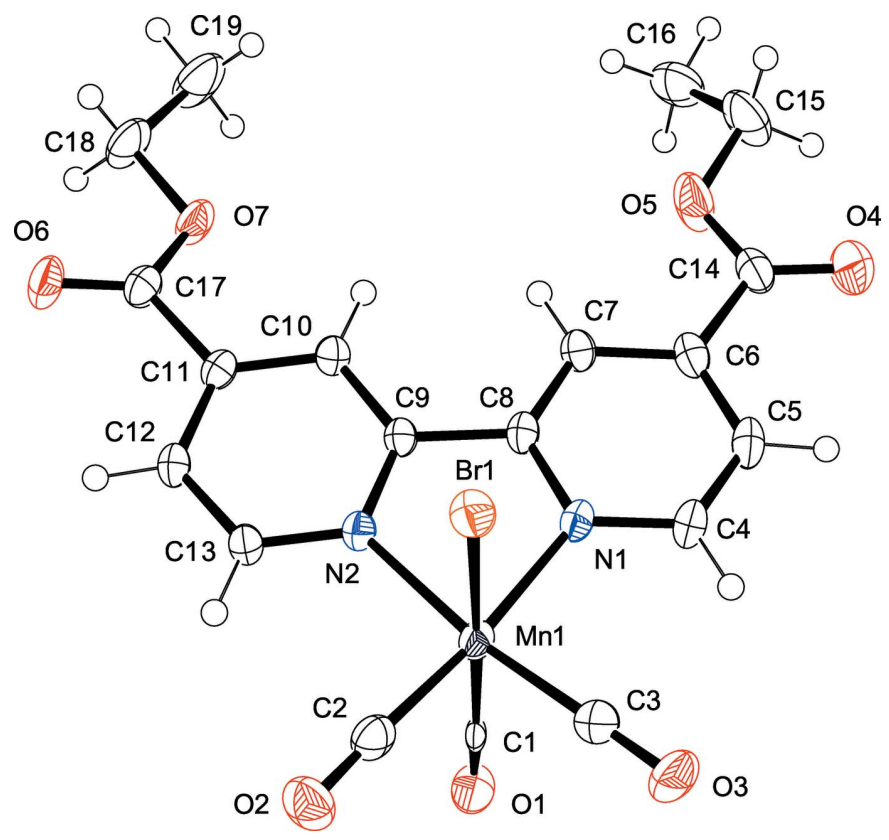

Figure 1

Molecular structure of I with atom labeling and displacement ellipsoids drawn at the $50 \%$ probability level.

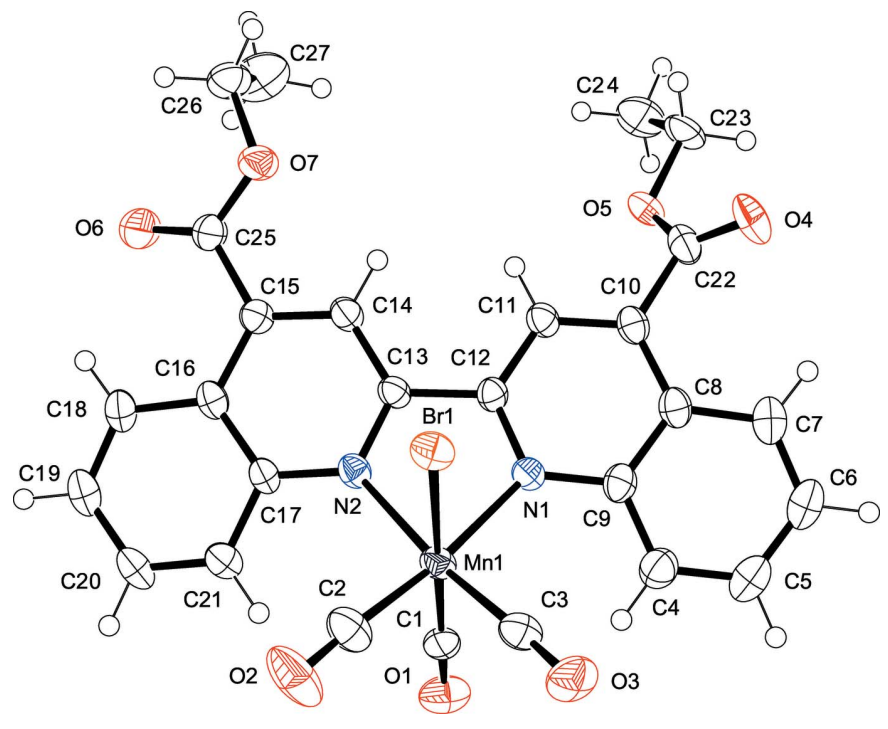

Figure 2

Molecular structure of II with atom labeling and displacement ellipsoids drawn at the $50 \%$ probability level.

$\mathrm{N} 2-\mathrm{C} 9)$ are $-169.17(15)$ and $168.81(14)^{\circ}$, respectively; the corresponding torsion angles in II $(\mathrm{C} 3-\mathrm{Mn} 1-\mathrm{N} 1-\mathrm{C} 12$ and $\mathrm{C} 2-\mathrm{Mn} 1-\mathrm{N} 2-\mathrm{C} 13)$ are $-147.52(16)$ and $147.08(17)^{\circ}$, respectively (Fig. 3). The large differences in torsion angles between $\mathbf{I}$ and $\mathbf{I I}$ are mainly due to steric hindrance between $\mathrm{H}$ atoms (H1 and $\mathrm{H} 10)$ in debqn, and the equatorial $\mathrm{CO}$ ligands $(\mathrm{C} 3 \equiv \mathrm{O} 3$ and $\mathrm{C} 2 \equiv \mathrm{O} 2)$. On the basis of similar steric hindrance, comparable torsion angles $[150.4(15)$ and $-150.7(5)^{\circ}$ ] have been also observed in the related $\mathrm{Re}^{\mathrm{I}}$ complex (Hallett et al., 2011).

Despite similar molecular skeletons, only II exhibits intramolecular hydrogen bonds between the ester group and the quinolyl ring (Table 2). The $\mathrm{C}-\mathrm{C}$ bond lengths of the coordinated pyridyl rings in $\mathbf{I}[\mathrm{C} 6-\mathrm{C} 7=1.395$ (3) $\AA$ and $\mathrm{C} 10-\mathrm{C} 11=1.392(3) \AA]$ are considerably longer than the corresponding one in II [C10-C11 = 1.364 (4) $\AA$ and C14$\mathrm{C} 15=1.368$ (4) $\mathrm{A}]$. This difference in structural parameters may eventually affect the intramolecular hydrogen-bond formation.

\section{Supramolecular features}

In the crystal structure of $\mathbf{I}$, complex molecules are linked by pairs of weak $\mathrm{C}-\mathrm{H} \cdots \mathrm{Br}$ hydrogen bonds (Table 1 ) and $\pi-\pi$ interactions $\left[C g 1 \cdots C g 2^{\mathrm{iii}}=3.683(1) \AA \AA^{\circ} C g 1\right.$ and $C g 2$ are the
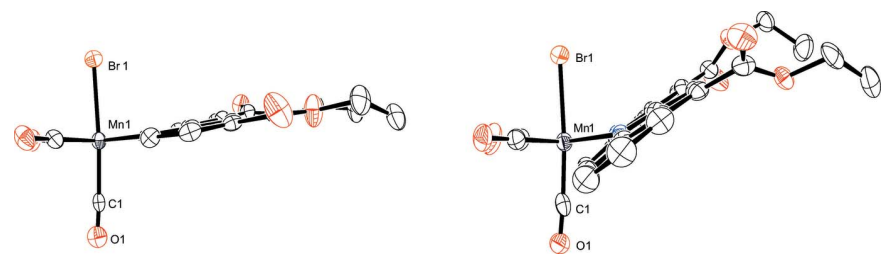

Figure 3

Side-on views of I (left) and II (right). H atoms are omitted for clarity. 
Table 1

Hydrogen-bond geometry $\left(\AA{ }^{\circ}\right)$ for $\mathbf{I}$.

\begin{tabular}{lllll}
\hline$D-\mathrm{H} \cdots A$ & $D-\mathrm{H}$ & $\mathrm{H} \cdots A$ & $D \cdots A$ & $D-\mathrm{H} \cdots A$ \\
\hline $\mathrm{C} 5-\mathrm{H} 2 \cdots \mathrm{Br}^{\mathrm{i}}{ }^{\mathrm{ii}}$ & 0.95 & 2.90 & $3.502(3)$ & 122 \\
$\mathrm{C} 13-\mathrm{H} 6 \cdots \mathrm{Br}^{\mathrm{ii}}$ & 0.95 & 2.78 & $3.537(3)$ & 138 \\
\hline
\end{tabular}

Symmetry codes: (i) $x+\frac{1}{2},-y+\frac{1}{2}, z$; (ii) $x-\frac{1}{2},-y+\frac{1}{2}, z$.

centroids of the N1/C4-C8 and N2/C9-C13 rings, respectively; symmetry code: (iii) $1-x,-y, 1-z]$, forming a threedimensional supramolecular structure (Fig. 4).

In the crystal structure of II, there are weak $\mathrm{C}-\mathrm{H} \cdots \mathrm{O}$ and $\mathrm{C}-\mathrm{H} \cdots \mathrm{Br}$ hydrogen-bonding interactions (Table 2) as well as the above-mentioned intramolecular hydrogen bonds. Additional $\pi-\pi$ contacts are observed $\left[C g 3 \cdots C g 4^{\text {iv }}=3.732\right.$ (2) $\AA$ and $C g 5 \cdots C g 6^{\text {iv }}=4.002$ (2) $\AA$; $C g 3, C g 4, C g 5$ and $C g 6$ are the centroids of the C4-C9, C16-C21, N1/C8-C12 and N2/C13C17 rings, respectively; symmetry code: (iv) $1-x, 1-y,-z]$. These interactions lead to the formation of a three-dimensional network structure (Fig. 5).

\section{Database survey}

With respect to manganese(I) complexes with a bidentate bipyridine derivative ligand $(\mathrm{N}-\mathrm{N})$ of the form fac$\left[\mathrm{MnBr}(\mathrm{CO})_{3}(\mathrm{~N}-\mathrm{N})\right]$, some structures have been reported (CSD refcode POKGAZ; Chakraborty et al., 2014a, FUMKOQ and FUMKUW; Henke et al., 2020, NIBSOJ; Lense et al., 2018, XUVMUY and XUVNAF; Walsh et al., 2015). However, no structures of bidentate biquinoline derivative-coordinated manganese(I) complexes have been reported; two structures of the corresponding rhenium(I) complexes have been determined by Hallett et al., 2011 (EBANEC) and Kurz et al., 2006 (XELXOC).

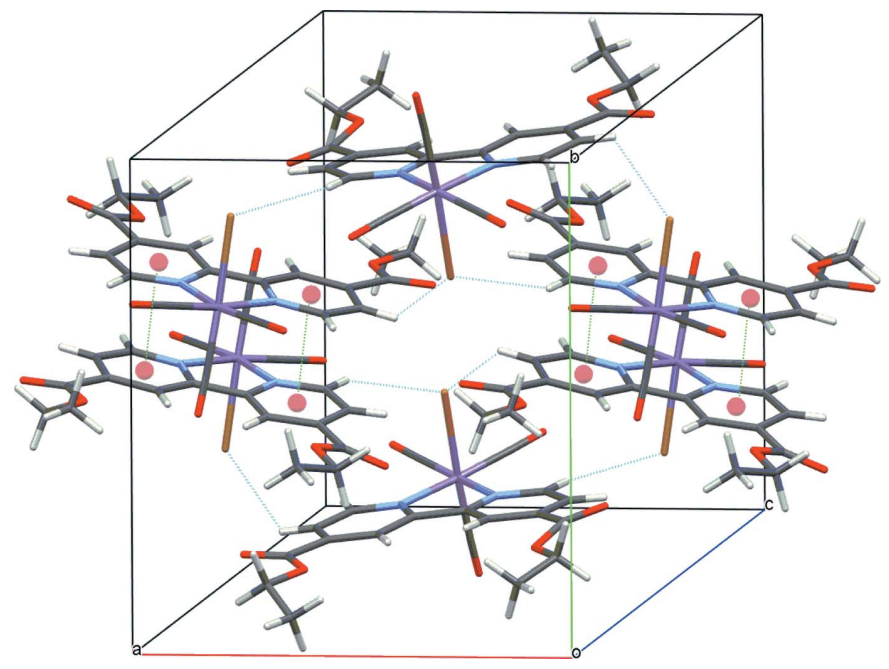

Figure 4

Crystal packing of $\mathbf{I}$ with $\mathrm{C}-\mathrm{H} \cdots \mathrm{Br}$ hydrogen bonds (blue) and $\pi-\pi$ contacts (green) shown as dashed lines; ring centroids are shown as red spheres.
Table 2

Hydrogen-bond geometry $\left(\AA,^{\circ}\right)$ for II.

\begin{tabular}{lllll}
\hline$D-\mathrm{H} \cdots A$ & $D-\mathrm{H}$ & $\mathrm{H} \cdots A$ & $D \cdots A$ & $D-\mathrm{H} \cdots A$ \\
\hline $\mathrm{C} 7-\mathrm{H} 4 \cdots \mathrm{O} 4$ & 0.95 & 2.44 & $3.040(4)$ & 121 \\
$\mathrm{C} 11-\mathrm{H} 5 \cdots \mathrm{Br} 1^{\mathrm{i}}$ & 0.95 & 2.92 & $3.789(3)$ & 153 \\
$\mathrm{C} 14-\mathrm{H} 6 \cdots \mathrm{O} 7$ & 0.95 & 2.33 & $2.659(3)$ & 100 \\
$\mathrm{C} 18-\mathrm{H} 7 \cdots \mathrm{O} 6$ & 0.95 & 2.25 & $2.883(5)$ & 124 \\
$\mathrm{C} 19-\mathrm{H} 8 \cdots 2^{\mathrm{ii}}$ & 0.95 & 2.47 & $3.373(4)$ & 160 \\
$\mathrm{C}^{\mathrm{iii}}-\mathrm{H} 9 \cdots$ O6 $^{\mathrm{iii}}$ & 0.95 & 2.51 & $3.383(4)$ & 153 \\
\hline
\end{tabular}

Symmetry codes: (i) $\quad-x,-y+1,-z ; \quad$ (ii) $\quad-x+1, y-\frac{1}{2},-z+\frac{1}{2}$; $\quad$ (iii) $-x+1, y+\frac{1}{2},-z+\frac{1}{2}$.

\section{Synthesis and crystallization}

The ligands, debpy and debqn, were prepared as described by Chandrasekharam et al. (2011) and Hoertz et al. (2006). The ligands were confirmed to be spectroscopically pure (by IR and ${ }^{1} \mathrm{H}$ NMR analyses).

Synthesis of I and II: Compounds I and II were handled and stored in the dark to minimize exposure to light. For the synthesis of $\mathbf{I},\left[\mathrm{MnBr}(\mathrm{CO})_{5}\right](31 \mathrm{mg}, 0.11 \mathrm{mmol})$ and debpy (33 mg, $0.11 \mathrm{mmol})$ were dissolved in $\mathrm{CHCl}_{3}(10 \mathrm{ml})$. The reaction mixture was stirred at $313 \mathrm{~K}$ for $14 \mathrm{~h}$ under $\mathrm{N}_{2}$. After the solvent was evaporated under reduced pressure, an excess of $\mathrm{Et}_{2} \mathrm{O}(30 \mathrm{ml})$ was added to the solution; then, the solution was allowed to stand at $253 \mathrm{~K}$ overnight. The resultant precipitate was collected by filtration, washed with $\mathrm{Et}_{2} \mathrm{O}$, and then dried under vacuum (37 mg yield, 64\%). Red crystals, suitable for the X-ray diffraction experiment, were grown by diffusion of $n$-hexane into an acetone solution of $\mathbf{I}$ for one week. FTIR ( $\mathrm{KBr}$ pellet): $\nu \mathrm{CO} / \mathrm{cm}^{-1}=2028,1918(b r)$ $(\mathrm{C} \equiv \mathrm{O}), 1730(\mathrm{C}=\mathrm{O})$. UV-vis $\left(\mathrm{CHCl}_{3}\right): \lambda / \mathrm{nm}\left(\varepsilon / \mathrm{M}^{-1} \mathrm{~cm}^{-1}\right)$ $=483$ (3700), 367 (4100), 318 (21000), 247 (24000).

A similar reaction between $\left[\mathrm{MnBr}(\mathrm{CO})_{5}\right] \quad(8 \mathrm{mg}$, $0.029 \mathrm{mmol})$ and debqn $(10 \mathrm{mg}, 0.026 \mathrm{mmol})$ for $20 \mathrm{~h}$ afforded II (11 mg yield, 66\%). Purple crystals, suitable for the X-ray diffraction experiment, were grown by diffusion of $n$-hexane into an acetone solution of II for one week. FTIR $(\mathrm{KBr}$ pellet): $\nu \mathrm{CO} / \mathrm{cm}^{-1}=2016,1942,1926(\mathrm{C} \equiv \mathrm{O}), 1725(\mathrm{C}=\mathrm{O})$. UV-vis $\left(\mathrm{CHCl}_{3}\right): \lambda / \mathrm{nm} \quad\left(\varepsilon / \mathrm{M}^{-1} \mathrm{~cm}^{-1}\right)=548(3200)$, 383 (19000), 276 (37000).

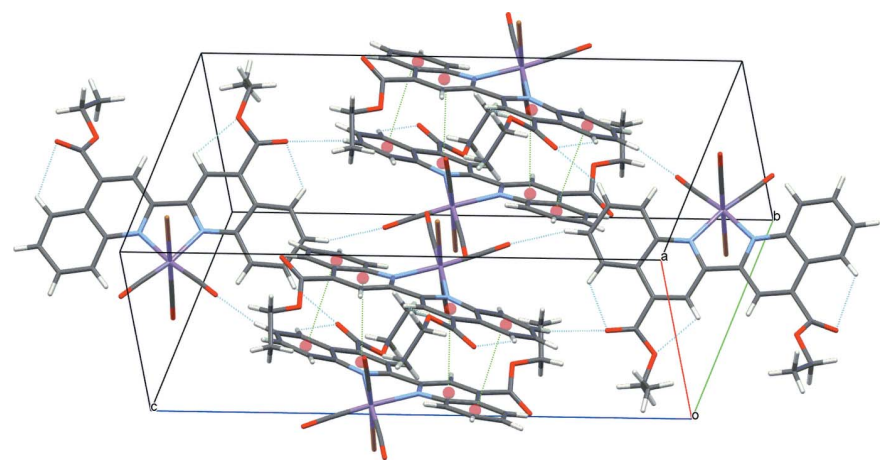

Figure 5

Crystal packing of II with $\mathrm{C}-\mathrm{H} \cdots \mathrm{Br}$ hydrogen bonds (blue) and $\pi-\pi$ contacts (green) shown as dashed lines; ring centroids are shown as red spheres. 
Table 3

Experimental details.

I

Crystal data

Chemical formula

$M_{\mathrm{r}}$

Crystal system, space group

Temperature $(\mathrm{K})$

$a, b, c(\AA)$

$\beta\left({ }^{\circ}\right)$

$V\left(\AA^{3}\right)$

Z

Radiation type

$\mu\left(\mathrm{mm}^{-1}\right)$

Crystal size (mm)

Data collection

Diffractometer

Absorption correction

$T_{\min }, T_{\max }$

No. of measured, independent and

observed $\left[F^{2}>2.0 \sigma\left(F^{2}\right)\right]$ reflections

$R_{\text {int }}$

$(\sin \theta / \lambda)_{\max }\left(\AA^{-1}\right)$

Refinemen

$R\left[F^{2}>2 \sigma\left(F^{2}\right)\right], w R\left(F^{2}\right), S$

No. of reflections

No. of parameters

$\mathrm{H}$-atom treatment

$\Delta \rho_{\max }, \Delta \rho_{\min }\left(\mathrm{e} \AA^{-3}\right)$

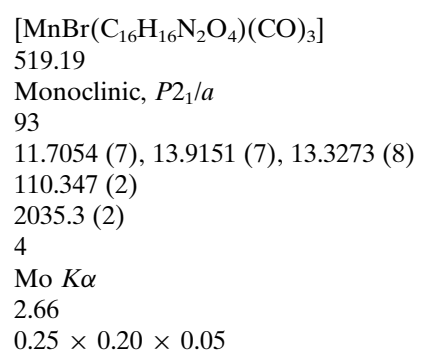

II

$\left[\mathrm{MnBr}\left(\mathrm{C}_{24} \mathrm{H}_{20} \mathrm{~N}_{2} \mathrm{O}_{4}\right)(\mathrm{CO})_{3}\right]$

619.31

Monoclinic, $P 2_{1} / c$

93

8.8953 (9), 12.0086 (13), 23.790 (3)

$95.794(2)$

$2528.3(5)$

4

Mo $K \alpha$

2.16

$0.20 \times 0.08 \times 0.05$

Rigaku Saturn724

Multi-scan (REQAB; Rigaku, 1998)

$0.730,0.875$

20542, 4653, 4050

0.029

0.650

Rigaku Saturn70

Multi-scan (REQAB; Rigaku, 1998)

$0.461,0.898$

25401, 5757, 4100

0.080

0.649

$0.037,0.098,1.07$

4653

273

$\mathrm{H}$-atom parameters constrained

$1.05,-0.56$
$0.046,0.134,1.05$

5757

345

$\mathrm{H}$-atom parameters constrained $0.79,-1.07$

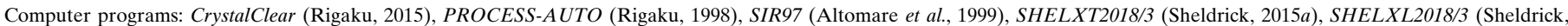
2015b), Mercury (Macrae et al., 2020), ORTEP-3 for Windows (Farrugia, 2012), CrystalStructure (Rigaku, 2019), PLATON (Spek, 2020) and publCIF (Westrip, 2010)

\section{Refinement}

Crystal data, data collection and structure refinement details are summarized in Table 3. All hydrogen atoms were placed at calculated positions $(\mathrm{C}-\mathrm{H}=0.95-0.99 \AA)$ and refined using a riding model with $U_{\text {iso }}(\mathrm{H})=1.2 U_{\text {eq }}(\mathrm{C})$.

\section{Funding information}

Funding for this research was provided by: Japan Society for the Promotion of Science (grant No. JP17K05799).

\section{References}

Altomare, A., Burla, M. C., Camalli, M., Cascarano, G. L., Giacovazzo, C., Guagliardi, A., Moliterni, A. G. G., Polidori, G. \& Spagna, R. (1999). J. Appl. Cryst. 32, 115-119.

Ardo, S. \& Meyer, G. J. (2009). Chem. Soc. Rev. 38, 115-164.

Bourrez, M., Molton, F., Chardon-Noblat, S. \& Deronzier, A. (2011). Angew. Chem. Int. Ed. 50, 9903-9906.

Chakraborty, I., Carrington, S. J. \& Mascharak, P. K. (2014a). ChemMedChem, 9, 1266-1274.

Chakraborty, I., Carrington, S. J. \& Mascharak, P. K. (2014b). Acc. Chem. Res. 47, 2603-2611.

Chandrasekharam, M., Srinivasarao, C., Suresh, T., Reddy, S. M., Raghavender, M., Rajkumar, G., Srinivasu, M. \& Reddy, P. Y. (2011). J. Chem. Sci. 123, 37-46.
Farrugia, L. J. (2012). J. Appl. Cryst. 45, 849-854.

Hallett, A. J. \& Pope, J. A. (2011). Inorg. Chem. Commun. 14, 16061608.

Henke, W. C., Otolski, C. J., Moore, W. N. G., Elles, C. G. \& Blakemore, J. D. (2020). Inorg. Chem. 59, 2178-2187.

Hoertz, P. G., Staniszewski, A., Marton, A., Higgins, G. T., Incarvito, C. D., Rheingold, A. L. \& Meyer, G. J. (2006). J. Am. Chem. Soc. 128, 8234-8245.

Kurz, P., Probst, B., Spingler, B. \& Alberto, R. (2006). Eur. J. Inorg. Chem. pp. 2966-2974.

Lense, S., Guzei, I. A., Andersen, J. \& Thao, K. C. (2018). Acta Cryst. E74, 731-736.

Macrae, C. F., Sovago, I., Cottrell, S. J., Galek, P. T. A., McCabe, P., Pidcock, E., Platings, M., Shields, G. P., Stevens, J. S., Towler, M. \& Wood, P. A. (2020). J. Appl. Cryst. 53, 226-235.

Motterlini, R., Clark, J. E., Foresti, R., Sarathchandra, P., Mann, B. E. \& Green, C. J. (2002). Circ. Res. 90, e17-e24.

Rigaku (1998). PROCESS-AUTO. Rigaku Corporation, Tokyo, Japan.

Rigaku (2015). CrystalClear. Rigaku Corporation, Tokyo, Japan.

Rigaku (2019). CrystalStructure. Rigaku Corporation, Tokyo, Japan.

Sheldrick, G. M. (2015a). Acta Cryst. A71, 3-8.

Sheldrick, G. M. (2015b). Acta Cryst. C71, 3-8.

Spek, A. L. (2020). Acta Cryst. E76, 1-11.

Walsh, J. J., Smith, C. L., Neri, G., Whitehead, G. F. S., Robertson, C. M. \& Cowan, A. J. (2015). Faraday Discuss. 183, 147-160.

Westrip, S. P. (2010). J. Appl. Cryst. 43, 920-925.

Zhang, L., He, R. \& Gu, H. C. (2006). Appl. Surf. Sci. 253, 2611-2617. 


\section{supporting information}

Acta Cryst. (2020). E76, 1433-1436 [https://doi.org/10.1107/S2056989020010750]

\section{Synthesis and crystal structures of manganese(I) carbonyl complexes bearing ester-substituted $\alpha$-diimine ligands}

\section{Takatoshi Kanno, Tsugiko Takase and Dai Oyama}

Computing details

Data collection: CrystalClear (Rigaku, 2015) for (I); PROCESS-AUTO (Rigaku, 1998) for (II). Cell refinement:

CrystalClear (Rigaku, 2015) for (I); PROCESS-AUTO (Rigaku, 1998) for (II). Data reduction: CrystalClear (Rigaku, 2015) for (I); PROCESS-AUTO (Rigaku, 1998) for (II). Program(s) used to solve structure: SIR97 (Altomare et al., 1999) for (I); SHELXT2018/3 (Sheldrick, 2015a) for (II). For both structures, program(s) used to refine structure:

SHELXL2018/3 (Sheldrick, 2015b); molecular graphics: Mercury (Macrae et al., 2020), ORTEP-3 for Windows (Farrugia, 2012). Software used to prepare material for publication: CrystalStructure (Rigaku, 2019), PLATON (Spek, 2020), publCIF (Westrip, 2010) for (I); CrystalStructure Rigaku, 2019), PLATON (Spek, 2020), publCIF (Westrip, 2010) for (II). fac-Bromidotricarbonyl (diethyl 2,2'-bipyridine-4,4'-dicarboxylate- $\kappa^{2} N, N^{\prime}$ ) manganese(I) (I)

Crystal data

$\left[\mathrm{MnBr}\left(\mathrm{C}_{16} \mathrm{H}_{16} \mathrm{~N}_{2} \mathrm{O}_{4}\right)(\mathrm{CO})_{3}\right]$

$M_{r}=519.19$

Monoclinic, $P 2_{1} / a$

$a=11.7054(7) \AA$

$b=13.9151(7) \AA$

$c=13.3273(8) \AA$

$\beta=110.347(2)^{\circ}$

$V=2035.3(2) \AA^{3}$

$Z=4$

Data collection

Rigaku Saturn724 diffractometer

Detector resolution: 28.626 pixels $\mathrm{mm}^{-1}$

$\omega$ scans

Absorption correction: multi-scan

(REQAB; Rigaku, 1998)

$T_{\text {min }}=0.730, T_{\text {max }}=0.875$

20542 measured reflections

Refinement

Refinement on $F^{2}$

$R\left[F^{2}>2 \sigma\left(F^{2}\right)\right]=0.037$

$w R\left(F^{2}\right)=0.098$

$S=1.07$

4653 reflections

273 parameters
$F(000)=1040.00$

$D_{\mathrm{x}}=1.694 \mathrm{Mg} \mathrm{m}^{-3}$

Mo $K \alpha$ radiation, $\lambda=0.71075 \AA$

Cell parameters from 5049 reflections

$\theta=3.2-27.5^{\circ}$

$\mu=2.66 \mathrm{~mm}^{-1}$

$T=93 \mathrm{~K}$

Block, red

$0.25 \times 0.20 \times 0.05 \mathrm{~mm}$

4653 independent reflections

4050 reflections with $F^{2}>2.0 \sigma\left(F^{2}\right)$

$R_{\text {int }}=0.029$

$\theta_{\text {max }}=27.5^{\circ}, \theta_{\min }=3.2^{\circ}$

$h=-15 \rightarrow 15$

$k=-18 \rightarrow 18$

$l=-16 \rightarrow 17$

0 restraints

Primary atom site location: structure-invariant direct methods

Secondary atom site location: difference Fourier map 
Hydrogen site location: inferred from neighbouring sites

H-atom parameters constrained

$$
\begin{aligned}
& w=1 /\left[\sigma^{2}\left(F_{\mathrm{o}}^{2}\right)+(0.055 P)^{2}+1.4779 P\right] \\
& \text { where } P=\left(F_{\mathrm{o}}^{2}+2 F_{\mathrm{c}}{ }^{2}\right) / 3 \\
& (\Delta / \sigma)_{\max }=0.001 \\
& \Delta \rho_{\max }=1.05 \mathrm{e} \AA^{-3} \\
& \Delta \rho_{\min }=-0.56 \mathrm{e} \AA^{-3}
\end{aligned}
$$

Special details

Geometry. All esds (except the esd in the dihedral angle between two 1.s. planes) are estimated using the full covariance matrix. The cell esds are taken into account individually in the estimation of esds in distances, angles and torsion angles; correlations between esds in cell parameters are only used when they are defined by crystal symmetry. An approximate (isotropic) treatment of cell esds is used for estimating esds involving l.s. planes.

\begin{tabular}{|c|c|c|c|c|}
\hline & $x$ & $y$ & $z$ & $U_{\text {iso }} * / U_{\text {eq }}$ \\
\hline Br1 & $0.61566(2)$ & $0.30862(2)$ & $0.74453(2)$ & $0.02885(10)$ \\
\hline Mn1 & $0.59428(3)$ & $0.13106(3)$ & $0.76285(3)$ & $0.02227(11)$ \\
\hline $\mathrm{O} 1$ & $0.56476(18)$ & $-0.07302(16)$ & $0.80205(17)$ & $0.0373(5)$ \\
\hline $\mathrm{O} 2$ & $0.4659(2)$ & 0.17993 (16) & $0.91224(17)$ & $0.0383(5)$ \\
\hline $\mathrm{O} 3$ & $0.82383(19)$ & $0.14080(17)$ & $0.94807(17)$ & $0.0445(6)$ \\
\hline $\mathrm{O} 4$ & $0.9317(2)$ & $0.0758(2)$ & $0.4214(2)$ & $0.0611(8)$ \\
\hline $\mathrm{O} 5$ & 0.74278 (19) & $0.0819(2)$ & $0.30486(17)$ & $0.0491(6)$ \\
\hline O6 & $0.06293(17)$ & $0.21138(15)$ & $0.31716(16)$ & $0.0348(5)$ \\
\hline $\mathrm{O} 7$ & $0.18898(17)$ & $0.15004(15)$ & $0.23897(15)$ & $0.0337(5)$ \\
\hline N1 & $0.67488(18)$ & $0.10977(15)$ & $0.65084(17)$ & $0.0225(4)$ \\
\hline $\mathrm{N} 2$ & $0.44701(18)$ & $0.13714(14)$ & $0.62383(16)$ & $0.0204(4)$ \\
\hline $\mathrm{C} 1$ & 0.57649 (19) & $-0.00038(19)$ & $0.78541(18)$ & $0.0184(5)$ \\
\hline $\mathrm{C} 2$ & $0.5130(2)$ & $0.1609(2)$ & $0.8528(2)$ & $0.0281(6)$ \\
\hline $\mathrm{C} 3$ & $0.7357(2)$ & $0.1367(2)$ & $0.8760(2)$ & $0.0300(6)$ \\
\hline $\mathrm{C} 4$ & $0.7938(2)$ & $0.09076(19)$ & $0.6709(2)$ & $0.0277(6)$ \\
\hline $\mathrm{H} 1$ & 0.846290 & 0.085051 & 0.743314 & $0.033^{*}$ \\
\hline $\mathrm{C} 5$ & $0.8427(2)$ & $0.07926(19)$ & $0.5918(2)$ & $0.0286(6)$ \\
\hline $\mathrm{H} 2$ & 0.926942 & 0.065380 & 0.609537 & $0.034^{*}$ \\
\hline C6 & $0.7678(2)$ & $0.08816(17)$ & $0.4859(2)$ & $0.0248(5)$ \\
\hline $\mathrm{C} 7$ & $0.6442(2)$ & $0.10676(17)$ & $0.4631(2)$ & $0.0227(5)$ \\
\hline $\mathrm{H} 3$ & 0.590464 & 0.112142 & 0.391116 & $0.027 *$ \\
\hline $\mathrm{C} 8$ & $0.6010(2)$ & $0.11726(17)$ & $0.5469(2)$ & $0.0212(5)$ \\
\hline C9 & $0.4718(2)$ & $0.13526(16)$ & $0.53200(19)$ & $0.0195(5)$ \\
\hline $\mathrm{C} 10$ & $0.3818(2)$ & $0.14873(17)$ & $0.4324(2)$ & $0.0218(5)$ \\
\hline H4 & 0.401900 & 0.149195 & 0.369186 & $0.026^{*}$ \\
\hline $\mathrm{C} 11$ & $0.2618(2)$ & $0.16152(17)$ & 0.42677 (19) & $0.0218(5)$ \\
\hline $\mathrm{C} 12$ & $0.2358(2)$ & $0.16067(18)$ & $0.5208(2)$ & $0.0228(5)$ \\
\hline H5 & 0.154399 & 0.167911 & 0.518946 & $0.027^{*}$ \\
\hline $\mathrm{C} 13$ & $0.3306(2)$ & $0.14909(17)$ & $0.6174(2)$ & $0.0219(5)$ \\
\hline H6 & 0.312656 & 0.149609 & 0.681603 & $0.026^{*}$ \\
\hline C14 & $0.8236(2)$ & $0.08021(19)$ & $0.4011(2)$ & $0.0300(6)$ \\
\hline
\end{tabular}

Refinement. Refinement was performed using all reflections. The weighted R-factor (wR) and goodness of fit (S) are based on $\mathrm{F}^{2}$. R-factor $(\mathrm{gt})$ are based on $\mathrm{F}$. The threshold expression of $\mathrm{F}^{2}>2.0 \operatorname{sigma}\left(\mathrm{F}^{2}\right)$ is used only for calculating $\mathrm{R}$ factor $(\mathrm{gt})$.

Fractional atomic coordinates and isotropic or equivalent isotropic displacement parameters $\left(\AA^{2}\right)$ 


$\begin{array}{lllll}\text { C15 } & 0.7888(3) & 0.0781(3) & 0.2161(3) & 0.0509(9) \\ \text { H7 } & 0.812429 & 0.143292 & 0.200741 & 0.061^{*} \\ \text { H8 } & 0.861458 & 0.036113 & 0.234873 & 0.061^{*} \\ \text { C16 } & 0.6927(3) & 0.0403(3) & 0.1227(3) & 0.0487(8) \\ \text { H9 } & 0.720196 & 0.040532 & 0.061186 & 0.058^{*} \\ \text { H10 } & 0.619799 & 0.080493 & 0.106964 & 0.058^{*} \\ \text { H11 } & 0.673358 & -0.025646 & 0.137198 & 0.058^{*} \\ \text { C17 } & 0.1594(2) & 0.17805(19) & 0.3228(2) & 0.0256(5) \\ \text { C18 } & 0.0934(3) & 0.1635(3) & 0.1343(2) & 0.0437(8) \\ \text { H12 } & 0.067516 & 0.231576 & 0.124367 & 0.052^{*} \\ \text { H13 } & 0.021624 & 0.123338 & 0.128791 & 0.052^{*} \\ \text { C19 } & 0.1454(4) & 0.1343(3) & 0.0510(2) & 0.0577(11) \\ \text { H14 } & 0.170249 & 0.066677 & 0.061456 & 0.069^{*} \\ \text { H15 } & 0.216324 & 0.174389 & 0.057323 & 0.069^{*} \\ \text { H16 } & 0.083590 & 0.142579 & -0.020197 & 0.069^{*}\end{array}$

Atomic displacement parameters $\left(\AA^{2}\right)$

\begin{tabular}{|c|c|c|c|c|c|c|}
\hline & $U^{11}$ & $U^{22}$ & $U^{33}$ & $U^{12}$ & $U^{13}$ & $U^{23}$ \\
\hline Br1 & $0.02690(15)$ & $0.02926(15)$ & $0.03069(16)$ & $-0.00070(10)$ & $0.01041(11)$ & $-0.00024(10)$ \\
\hline Mn1 & 0.01673 (19) & $0.0283(2)$ & 0.01915 (19) & $0.00051(14)$ & $0.00286(14)$ & $-0.00025(14)$ \\
\hline O1 & $0.0297(11)$ & $0.0444(13)$ & $0.0364(11)$ & $0.0040(9)$ & $0.0096(9)$ & $0.0013(9)$ \\
\hline $\mathrm{O} 2$ & $0.0380(12)$ & $0.0490(13)$ & $0.0330(11)$ & $-0.0006(9)$ & $0.0187(10)$ & $-0.0064(9)$ \\
\hline $\mathrm{O} 3$ & $0.0273(11)$ & $0.0608(15)$ & $0.0333(11)$ & $-0.0066(10)$ & $-0.0047(9)$ & $0.0106(10)$ \\
\hline $\mathrm{O} 4$ & $0.0359(13)$ & $0.099(2)$ & $0.0583(16)$ & $0.0226(13)$ & $0.0288(12)$ & $0.0239(15)$ \\
\hline $\mathrm{O5}$ & $0.0283(11)$ & 0.0907 (19) & $0.0335(11)$ & $-0.0039(11)$ & $0.0173(9)$ & $-0.0114(12)$ \\
\hline O6 & $0.0197(9)$ & $0.0471(12)$ & $0.0318(11)$ & $0.0090(8)$ & $0.0015(8)$ & $-0.0025(9)$ \\
\hline $\mathrm{O} 7$ & $0.0248(10)$ & $0.0503(12)$ & $0.0200(9)$ & $0.0118(8)$ & $0.0003(7)$ & $0.0020(8)$ \\
\hline N1 & $0.0160(9)$ & $0.0241(10)$ & $0.0241(10)$ & $0.0007(8)$ & $0.0027(8)$ & $-0.0001(8)$ \\
\hline N2 & $0.0164(9)$ & $0.0221(10)$ & $0.0215(10)$ & $-0.0004(7)$ & $0.0052(8)$ & $-0.0011(8)$ \\
\hline $\mathrm{C} 1$ & $0.0063(9)$ & $0.0344(14)$ & $0.0133(10)$ & $0.0023(9)$ & $0.0021(8)$ & $-0.0008(9)$ \\
\hline $\mathrm{C} 2$ & $0.0232(13)$ & $0.0321(13)$ & $0.0237(13)$ & $-0.0027(10)$ & $0.0015(11)$ & $0.0001(10)$ \\
\hline $\mathrm{C} 3$ & $0.0273(13)$ & $0.0344(14)$ & $0.0278(13)$ & $-0.0011(11)$ & $0.0089(11)$ & $0.0042(11)$ \\
\hline $\mathrm{C} 4$ & $0.0167(11)$ & $0.0316(13)$ & 0.0308 (13) & $0.0030(10)$ & $0.0033(10)$ & $-0.0014(11)$ \\
\hline $\mathrm{C} 5$ & $0.0164(11)$ & $0.0268(13)$ & $0.0410(15)$ & $0.0020(9)$ & $0.0080(11)$ & -0.0020 \\
\hline C6 & $0.0214(12)$ & $0.0197(12)$ & $0.0361(14)$ & $-0.0015(9)$ & $0.0136(11)$ & $-0.0030(10)$ \\
\hline $\mathrm{C} 7$ & $0.0192(11)$ & $0.0231(12)$ & $0.0258(12)$ & $-0.0022(9)$ & $0.0076(10)$ & $-0.0033(9)$ \\
\hline $\mathrm{C} 8$ & $0.0153(11)$ & $0.0210(11)$ & 0.0257 (12) & $-0.0008(9)$ & $0.0051(9)$ & $-0.0019(9)$ \\
\hline C9 & $0.0160(11)$ & $0.0198(11)$ & 0.0223 (11) & $-0.0012(8)$ & $0.0060(9)$ & $-0.0014(9)$ \\
\hline $\mathrm{C} 10$ & $0.0195(11)$ & $0.0227(12)$ & $0.0232(12)$ & $0.0013(9)$ & $0.0074(10)$ & $-0.0013(9)$ \\
\hline C11 & 0.0185 (11) & $0.0217(11)$ & $0.0217(12)$ & $0.0006(9)$ & $0.0026(9)$ & $-0.0008(9)$ \\
\hline $\mathrm{C} 12$ & $0.0151(11)$ & $0.0246(12)$ & $0.0275(13)$ & $-0.0004(9)$ & $0.0058(10)$ & $-0.0018(10)$ \\
\hline C13 & 0.0177 (11) & $0.0245(12)$ & $0.0238(12)$ & $0.0000(9)$ & $0.0078(9)$ & $-0.0011(9)$ \\
\hline $\mathrm{C} 14$ & $0.0264(14)$ & $0.0261(13)$ & $0.0433(16)$ & $0.0003(10)$ & 0.0194 (12) & $0.0022(11)$ \\
\hline $\mathrm{C} 15$ & 0.0442 (19) & $0.076(3)$ & 0.0455 (19) & $-0.0077(17)$ & $0.0318(16)$ & $-0.0074(17)$ \\
\hline $\mathrm{C} 16$ & $0.054(2)$ & $0.065(2)$ & $0.0344(17)$ & $-0.0040(17)$ & $0.0246(15)$ & $0.0039(16)$ \\
\hline $\mathrm{C} 17$ & 0.0202 (12) & $0.0275(13)$ & $0.0252(13)$ & $0.0000(10)$ & $0.0028(10)$ & $-0.0001(10)$ \\
\hline $\mathrm{C} 18$ & $0.0335(16)$ & $0.066(2)$ & $0.0220(14)$ & $0.0164(15)$ & $-0.0026(12)$ & $0.0040(14)$ \\
\hline
\end{tabular}




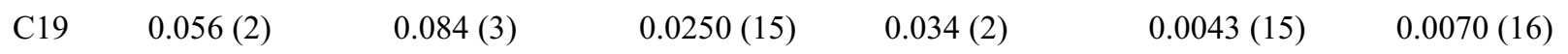

Geometric parameters $(\AA, \stackrel{o}{)}$

\begin{tabular}{|c|c|c|c|}
\hline $\mathrm{Br} 1-\mathrm{Mn} 1$ & $2.5038(5)$ & $\mathrm{C} 6-\mathrm{C} 14$ & $1.493(4)$ \\
\hline $\mathrm{Mn} 1-\mathrm{C} 3$ & $1.812(3)$ & $\mathrm{C} 7-\mathrm{C} 8$ & $1.385(4)$ \\
\hline $\mathrm{Mn} 1-\mathrm{C} 2$ & $1.819(3)$ & $\mathrm{C} 7-\mathrm{H} 3$ & 0.9500 \\
\hline $\mathrm{Mn} 1-\mathrm{C} 1$ & $1.877(3)$ & $\mathrm{C} 8-\mathrm{C} 9$ & $1.477(3)$ \\
\hline $\mathrm{Mn} 1-\mathrm{N} 1$ & $2.046(2)$ & $\mathrm{C} 9-\mathrm{C} 10$ & $1.391(3)$ \\
\hline $\mathrm{Mn} 1-\mathrm{N} 2$ & $2.047(2)$ & $\mathrm{C} 10-\mathrm{C} 11$ & $1.392(3)$ \\
\hline $\mathrm{O} 1-\mathrm{C} 1$ & $1.054(3)$ & $\mathrm{C} 10-\mathrm{H} 4$ & 0.9500 \\
\hline $\mathrm{O} 2-\mathrm{C} 2$ & $1.142(3)$ & $\mathrm{C} 11-\mathrm{C} 12$ & $1.389(4)$ \\
\hline $\mathrm{O} 3-\mathrm{C} 3$ & $1.141(3)$ & $\mathrm{C} 11-\mathrm{C} 17$ & $1.502(3)$ \\
\hline $\mathrm{O} 4-\mathrm{C} 14$ & $1.200(3)$ & $\mathrm{C} 12-\mathrm{C} 13$ & $1.386(3)$ \\
\hline $\mathrm{O} 5-\mathrm{C} 14$ & $1.303(4)$ & $\mathrm{C} 12-\mathrm{H} 5$ & 0.9500 \\
\hline $\mathrm{O} 5-\mathrm{C} 15$ & $1.461(4)$ & $\mathrm{C} 13-\mathrm{H} 6$ & 0.9500 \\
\hline $\mathrm{O} 6-\mathrm{C} 17$ & $1.199(3)$ & $\mathrm{C} 15-\mathrm{C} 16$ & $1.455(5)$ \\
\hline $\mathrm{O} 7-\mathrm{C} 17$ & $1.337(3)$ & $\mathrm{C} 15-\mathrm{H} 7$ & 0.9900 \\
\hline $\mathrm{O} 7-\mathrm{C} 18$ & $1.466(3)$ & $\mathrm{C} 15-\mathrm{H} 8$ & 0.9900 \\
\hline $\mathrm{N} 1-\mathrm{C} 4$ & $1.350(3)$ & $\mathrm{C} 16-\mathrm{H} 9$ & 0.9800 \\
\hline $\mathrm{N} 1-\mathrm{C} 8$ & $1.358(3)$ & $\mathrm{C} 16-\mathrm{H} 10$ & 0.9800 \\
\hline $\mathrm{N} 2-\mathrm{C} 13$ & $1.345(3)$ & C16-H11 & 0.9800 \\
\hline N2-C9 & $1.353(3)$ & $\mathrm{C} 18-\mathrm{C} 19$ & $1.495(5)$ \\
\hline $\mathrm{C} 4-\mathrm{C} 5$ & $1.373(4)$ & $\mathrm{C} 18-\mathrm{H} 12$ & 0.9900 \\
\hline $\mathrm{C} 4-\mathrm{H} 1$ & 0.9500 & $\mathrm{C} 18-\mathrm{H} 13$ & 0.9900 \\
\hline $\mathrm{C} 5-\mathrm{C} 6$ & $1.384(4)$ & C19-H14 & 0.9800 \\
\hline $\mathrm{C} 5-\mathrm{H} 2$ & 0.9500 & C19-H15 & 0.9800 \\
\hline $\mathrm{C} 6-\mathrm{C} 7$ & $1.395(3)$ & C19-H16 & 0.9800 \\
\hline $\mathrm{C} 3-\mathrm{Mn} 1-\mathrm{C} 2$ & $88.72(12)$ & $\mathrm{C} 10-\mathrm{C} 9-\mathrm{C} 8$ & $123.5(2)$ \\
\hline $\mathrm{C} 3-\mathrm{Mn} 1-\mathrm{C} 1$ & $91.66(11)$ & $\mathrm{C} 9-\mathrm{C} 10-\mathrm{C} 11$ & $118.9(2)$ \\
\hline $\mathrm{C} 2-\mathrm{Mn} 1-\mathrm{C} 1$ & $90.24(11)$ & $\mathrm{C} 9-\mathrm{C} 10-\mathrm{H} 4$ & 120.5 \\
\hline $\mathrm{C} 3-\mathrm{Mn} 1-\mathrm{N} 1$ & $95.38(11)$ & $\mathrm{C} 11-\mathrm{C} 10-\mathrm{H} 4$ & 120.5 \\
\hline $\mathrm{C} 2-\mathrm{Mn} 1-\mathrm{N} 1$ & $173.53(10)$ & $\mathrm{C} 12-\mathrm{C} 11-\mathrm{C} 10$ & $119.0(2)$ \\
\hline $\mathrm{C} 1-\mathrm{Mn} 1-\mathrm{N} 1$ & $94.63(9)$ & $\mathrm{C} 12-\mathrm{C} 11-\mathrm{C} 17$ & $118.6(2)$ \\
\hline $\mathrm{C} 3-\mathrm{Mn} 1-\mathrm{N} 2$ & $171.65(11)$ & $\mathrm{C} 10-\mathrm{C} 11-\mathrm{C} 17$ & $122.4(2)$ \\
\hline $\mathrm{C} 2-\mathrm{Mn} 1-\mathrm{N} 2$ & $96.79(10)$ & $\mathrm{C} 13-\mathrm{C} 12-\mathrm{C} 11$ & $118.9(2)$ \\
\hline $\mathrm{C} 1-\mathrm{Mn} 1-\mathrm{N} 2$ & $94.58(9)$ & $\mathrm{C} 13-\mathrm{C} 12-\mathrm{H} 5$ & 120.6 \\
\hline $\mathrm{N} 1-\mathrm{Mn} 1-\mathrm{N} 2$ & $78.60(8)$ & $\mathrm{C} 11-\mathrm{C} 12-\mathrm{H} 5$ & 120.6 \\
\hline $\mathrm{C} 3-\mathrm{Mn} 1-\mathrm{Br} 1$ & $86.86(9)$ & $\mathrm{N} 2-\mathrm{C} 13-\mathrm{C} 12$ & $122.7(2)$ \\
\hline $\mathrm{C} 2-\mathrm{Mn} 1-\mathrm{Br} 1$ & $86.10(9)$ & $\mathrm{N} 2-\mathrm{C} 13-\mathrm{H} 6$ & 118.7 \\
\hline $\mathrm{C} 1-\mathrm{Mn} 1-\mathrm{Br} 1$ & $176.08(7)$ & $\mathrm{C} 12-\mathrm{C} 13-\mathrm{H} 6$ & 118.7 \\
\hline $\mathrm{N} 1-\mathrm{Mn} 1-\mathrm{Br} 1$ & $89.12(6)$ & $\mathrm{O} 4-\mathrm{C} 14-\mathrm{O} 5$ & $124.8(3)$ \\
\hline $\mathrm{N} 2-\mathrm{Mn} 1-\mathrm{Br} 1$ & $87.26(6)$ & $\mathrm{O} 4-\mathrm{C} 14-\mathrm{C} 6$ & $122.6(3)$ \\
\hline $\mathrm{C} 14-\mathrm{O} 5-\mathrm{C} 15$ & $116.8(2)$ & $\mathrm{O} 5-\mathrm{C} 14-\mathrm{C} 6$ & $112.6(2)$ \\
\hline $\mathrm{C} 17-\mathrm{O} 7-\mathrm{C} 18$ & $115.1(2)$ & $\mathrm{C} 16-\mathrm{C} 15-\mathrm{O} 5$ & $108.2(3)$ \\
\hline $\mathrm{C} 4-\mathrm{N} 1-\mathrm{C} 8$ & $117.7(2)$ & $\mathrm{C} 16-\mathrm{C} 15-\mathrm{H} 7$ & 110.1 \\
\hline $\mathrm{C} 4-\mathrm{N} 1-\mathrm{Mn} 1$ & $126.13(17)$ & $\mathrm{O} 5-\mathrm{C} 15-\mathrm{H} 7$ & 110.1 \\
\hline
\end{tabular}




\begin{tabular}{|c|c|c|c|}
\hline $\mathrm{C} 8-\mathrm{N} 1-\mathrm{Mn} 1$ & $116.18(16)$ & $\mathrm{C} 16-\mathrm{C} 15-\mathrm{H} 8$ & 110.1 \\
\hline $\mathrm{C} 13-\mathrm{N} 2-\mathrm{C} 9$ & $118.4(2)$ & $\mathrm{O} 5-\mathrm{C} 15-\mathrm{H} 8$ & 110.1 \\
\hline $\mathrm{C} 13-\mathrm{N} 2-\mathrm{Mn} 1$ & $125.35(17)$ & $\mathrm{H} 7-\mathrm{C} 15-\mathrm{H} 8$ & 108.4 \\
\hline $\mathrm{C} 9-\mathrm{N} 2-\mathrm{Mn} 1$ & $116.12(15)$ & $\mathrm{C} 15-\mathrm{C} 16-\mathrm{H} 9$ & 109.5 \\
\hline $\mathrm{O} 1-\mathrm{C} 1-\mathrm{Mn} 1$ & $176.5(2)$ & $\mathrm{C} 15-\mathrm{C} 16-\mathrm{H} 10$ & 109.5 \\
\hline $\mathrm{O} 2-\mathrm{C} 2-\mathrm{Mn} 1$ & $177.5(2)$ & $\mathrm{H} 9-\mathrm{C} 16-\mathrm{H} 10$ & 109.5 \\
\hline $\mathrm{O} 3-\mathrm{C} 3-\mathrm{Mn} 1$ & $179.0(3)$ & $\mathrm{C} 15-\mathrm{C} 16-\mathrm{H} 11$ & 109.5 \\
\hline $\mathrm{N} 1-\mathrm{C} 4-\mathrm{C} 5$ & $123.2(2)$ & $\mathrm{H} 9-\mathrm{C} 16-\mathrm{H} 11$ & 109.5 \\
\hline $\mathrm{N} 1-\mathrm{C} 4-\mathrm{H} 1$ & 118.4 & $\mathrm{H} 10-\mathrm{C} 16-\mathrm{H} 11$ & 109.5 \\
\hline $\mathrm{C} 5-\mathrm{C} 4-\mathrm{H} 1$ & 118.4 & $\mathrm{O} 6-\mathrm{C} 17-\mathrm{O} 7$ & $124.9(2)$ \\
\hline $\mathrm{C} 4-\mathrm{C} 5-\mathrm{C} 6$ & $119.1(2)$ & $\mathrm{O} 6-\mathrm{C} 17-\mathrm{C} 11$ & $123.3(3)$ \\
\hline $\mathrm{C} 4-\mathrm{C} 5-\mathrm{H} 2$ & 120.4 & $\mathrm{O} 7-\mathrm{C} 17-\mathrm{C} 11$ & $111.7(2)$ \\
\hline $\mathrm{C} 6-\mathrm{C} 5-\mathrm{H} 2$ & 120.4 & $\mathrm{O} 7-\mathrm{C} 18-\mathrm{C} 19$ & $107.4(2)$ \\
\hline $\mathrm{C} 5-\mathrm{C} 6-\mathrm{C} 7$ & $118.7(2)$ & $\mathrm{O} 7-\mathrm{C} 18-\mathrm{H} 12$ & 110.2 \\
\hline $\mathrm{C} 5-\mathrm{C} 6-\mathrm{C} 14$ & $118.4(2)$ & $\mathrm{C} 19-\mathrm{C} 18-\mathrm{H} 12$ & 110.2 \\
\hline $\mathrm{C} 7-\mathrm{C} 6-\mathrm{C} 14$ & $122.9(2)$ & $\mathrm{O} 7-\mathrm{C} 18-\mathrm{H} 13$ & 110.2 \\
\hline $\mathrm{C} 8-\mathrm{C} 7-\mathrm{C} 6$ & $119.1(2)$ & $\mathrm{C} 19-\mathrm{C} 18-\mathrm{H} 13$ & 110.2 \\
\hline $\mathrm{C} 8-\mathrm{C} 7-\mathrm{H} 3$ & 120.5 & $\mathrm{H} 12-\mathrm{C} 18-\mathrm{H} 13$ & 108.5 \\
\hline $\mathrm{C} 6-\mathrm{C} 7-\mathrm{H} 3$ & 120.5 & $\mathrm{C} 18-\mathrm{C} 19-\mathrm{H} 14$ & 109.5 \\
\hline $\mathrm{N} 1-\mathrm{C} 8-\mathrm{C} 7$ & $122.2(2)$ & $\mathrm{C} 18-\mathrm{C} 19-\mathrm{H} 15$ & 109.5 \\
\hline $\mathrm{N} 1-\mathrm{C} 8-\mathrm{C} 9$ & $114.2(2)$ & $\mathrm{H} 14-\mathrm{C} 19-\mathrm{H} 15$ & 109.5 \\
\hline $\mathrm{C} 7-\mathrm{C} 8-\mathrm{C} 9$ & $123.6(2)$ & $\mathrm{C} 18-\mathrm{C} 19-\mathrm{H} 16$ & 109.5 \\
\hline $\mathrm{N} 2-\mathrm{C} 9-\mathrm{C} 10$ & $122.1(2)$ & $\mathrm{H} 14-\mathrm{C} 19-\mathrm{H} 16$ & 109.5 \\
\hline $\mathrm{N} 2-\mathrm{C} 9-\mathrm{C} 8$ & $114.4(2)$ & $\mathrm{H} 15-\mathrm{C} 19-\mathrm{H} 16$ & 109.5 \\
\hline $\mathrm{C} 8-\mathrm{N} 1-\mathrm{C} 4-\mathrm{C} 5$ & $0.3(4)$ & $\mathrm{C} 8-\mathrm{C} 9-\mathrm{C} 10-\mathrm{C} 11$ & $177.5(2)$ \\
\hline $\mathrm{Mn} 1-\mathrm{N} 1-\mathrm{C} 4-\mathrm{C} 5$ & $-178.5(2)$ & $\mathrm{C} 9-\mathrm{C} 10-\mathrm{C} 11-\mathrm{C} 12$ & $0.2(4)$ \\
\hline $\mathrm{N} 1-\mathrm{C} 4-\mathrm{C} 5-\mathrm{C} 6$ & $0.5(4)$ & $\mathrm{C} 9-\mathrm{C} 10-\mathrm{C} 11-\mathrm{C} 17$ & $179.0(2)$ \\
\hline $\mathrm{C} 4-\mathrm{C} 5-\mathrm{C} 6-\mathrm{C} 7$ & $-1.2(4)$ & $\mathrm{C} 10-\mathrm{C} 11-\mathrm{C} 12-\mathrm{C} 13$ & $1.3(4)$ \\
\hline $\mathrm{C} 4-\mathrm{C} 5-\mathrm{C} 6-\mathrm{C} 14$ & $177.1(2)$ & $\mathrm{C} 17-\mathrm{C} 11-\mathrm{C} 12-\mathrm{C} 13$ & $-177.6(2)$ \\
\hline $\mathrm{C} 5-\mathrm{C} 6-\mathrm{C} 7-\mathrm{C} 8$ & $1.1(4)$ & $\mathrm{C} 9-\mathrm{N} 2-\mathrm{C} 13-\mathrm{C} 12$ & $-0.8(4)$ \\
\hline $\mathrm{C} 14-\mathrm{C} 6-\mathrm{C} 7-\mathrm{C} 8$ & $-177.1(2)$ & $\mathrm{Mn} 1-\mathrm{N} 2-\mathrm{C} 13-\mathrm{C} 12$ & $174.46(18)$ \\
\hline $\mathrm{C} 4-\mathrm{N} 1-\mathrm{C} 8-\mathrm{C} 7$ & $-0.5(4)$ & $\mathrm{C} 11-\mathrm{C} 12-\mathrm{C} 13-\mathrm{N} 2$ & $-1.0(4)$ \\
\hline $\mathrm{Mn} 1-\mathrm{N} 1-\mathrm{C} 8-\mathrm{C} 7$ & $178.47(18)$ & $\mathrm{C} 15-\mathrm{O} 5-\mathrm{C} 14-\mathrm{O} 4$ & $0.0(5)$ \\
\hline $\mathrm{C} 4-\mathrm{N} 1-\mathrm{C} 8-\mathrm{C} 9$ & $178.3(2)$ & $\mathrm{C} 15-\mathrm{O} 5-\mathrm{C} 14-\mathrm{C} 6$ & $177.8(3)$ \\
\hline $\mathrm{Mn} 1-\mathrm{N} 1-\mathrm{C} 8-\mathrm{C} 9$ & $-2.8(3)$ & $\mathrm{C} 5-\mathrm{C} 6-\mathrm{C} 14-\mathrm{O} 4$ & $-7.4(4)$ \\
\hline $\mathrm{C} 6-\mathrm{C} 7-\mathrm{C} 8-\mathrm{N} 1$ & $-0.2(4)$ & $\mathrm{C} 7-\mathrm{C} 6-\mathrm{C} 14-\mathrm{O} 4$ & $170.8(3)$ \\
\hline $\mathrm{C} 6-\mathrm{C} 7-\mathrm{C} 8-\mathrm{C} 9$ & $-178.8(2)$ & $\mathrm{C} 5-\mathrm{C} 6-\mathrm{C} 14-\mathrm{O} 5$ & $174.7(2)$ \\
\hline $\mathrm{C} 13-\mathrm{N} 2-\mathrm{C} 9-\mathrm{C} 10$ & $2.4(3)$ & $\mathrm{C} 7-\mathrm{C} 6-\mathrm{C} 14-\mathrm{O} 5$ & $-7.1(4)$ \\
\hline $\mathrm{Mn} 1-\mathrm{N} 2-\mathrm{C} 9-\mathrm{C} 10$ & $-173.32(18)$ & $\mathrm{C} 14-\mathrm{O} 5-\mathrm{C} 15-\mathrm{C} 16$ & $156.1(3)$ \\
\hline $\mathrm{C} 13-\mathrm{N} 2-\mathrm{C} 9-\mathrm{C} 8$ & $-177.2(2)$ & $\mathrm{C} 18-\mathrm{O} 7-\mathrm{C} 17-\mathrm{O} 6$ & $0.5(4)$ \\
\hline $\mathrm{Mn} 1-\mathrm{N} 2-\mathrm{C} 9-\mathrm{C} 8$ & $7.0(3)$ & $\mathrm{C} 18-\mathrm{O} 7-\mathrm{C} 17-\mathrm{C} 11$ & $179.7(2)$ \\
\hline $\mathrm{N} 1-\mathrm{C} 8-\mathrm{C} 9-\mathrm{N} 2$ & $-2.8(3)$ & $\mathrm{C} 12-\mathrm{C} 11-\mathrm{C} 17-\mathrm{O} 6$ & $17.2(4)$ \\
\hline $\mathrm{C} 7-\mathrm{C} 8-\mathrm{C} 9-\mathrm{N} 2$ & $175.9(2)$ & $\mathrm{C} 10-\mathrm{C} 11-\mathrm{C} 17-\mathrm{O} 6$ & $-161.6(3)$ \\
\hline $\mathrm{N} 1-\mathrm{C} 8-\mathrm{C} 9-\mathrm{C} 10$ & $177.6(2)$ & $\mathrm{C} 12-\mathrm{C} 11-\mathrm{C} 17-\mathrm{O} 7$ & $-162.0(2)$ \\
\hline $\mathrm{C} 7-\mathrm{C} 8-\mathrm{C} 9-\mathrm{C} 10$ & $-3.7(4)$ & $\mathrm{C} 10-\mathrm{C} 11-\mathrm{C} 17-\mathrm{O} 7$ & $19.2(3)$ \\
\hline $\mathrm{N} 2-\mathrm{C} 9-\mathrm{C} 10-\mathrm{C} 11$ & $-2.1(4)$ & $\mathrm{C} 17-\mathrm{O} 7-\mathrm{C} 18-\mathrm{C} 19$ & $176.5(3)$ \\
\hline
\end{tabular}


Hydrogen-bond geometry $\left(\AA,{ }^{\circ}\right)$

\begin{tabular}{lllll}
\hline$D-\mathrm{H} \cdots A$ & $D-\mathrm{H}$ & $\mathrm{H} \cdots A$ & $D \cdots A$ & $D-\mathrm{H} \cdots A$ \\
\hline $\mathrm{C} 5-\mathrm{H} 2 \cdots \mathrm{Br}^{\mathrm{i}}$ & 0.95 & 2.90 & $3.502(3)$ & 122 \\
$\mathrm{C} 13-\mathrm{H} 6 \cdots \mathrm{Br}^{1}{ }^{\mathrm{ii}}$ & 0.95 & 2.78 & $3.537(3)$ & 138 \\
\hline
\end{tabular}

Symmetry codes: (i) $x+1 / 2,-y+1 / 2, z$; (ii) $x-1 / 2,-y+1 / 2, z$.

fac-Bromidotricarbonyl(diethyl 2,2'-biquinoline-4,4'-dicarboxylate- $\left.\kappa^{2} N, N^{\prime}\right)$ manganese(I) (II)

Crystal data

$\left[\mathrm{MnBr}\left(\mathrm{C}_{24} \mathrm{H}_{20} \mathrm{~N}_{2} \mathrm{O}_{4}\right)(\mathrm{CO})_{3}\right]$

$M_{r}=619.31$

Monoclinic, $P 2{ }_{1} / c$

$a=8.8953(9) \AA$

$b=12.0086(13) \AA$

$c=23.790(3) \AA$

$\beta=95.794(2)^{\circ}$

$V=2528.3(5) \AA^{3}$

$Z=4$

\section{Data collection}

Rigaku Saturn70 diffractometer

Detector resolution: 7.143 pixels $\mathrm{mm}^{-1}$

$\omega$ scans

Absorption correction: multi-scan

(REQAB; Rigaku, 1998)

$T_{\min }=0.461, T_{\max }=0.898$

25401 measured reflections

Refinement

Refinement on $F^{2}$

$R\left[F^{2}>2 \sigma\left(F^{2}\right)\right]=0.046$

$w R\left(F^{2}\right)=0.134$

$S=1.05$

5757 reflections

345 parameters

0 restraints

Primary atom site location: structure-invariant

direct methods
$F(000)=1248.00$

$D_{\mathrm{x}}=1.627 \mathrm{Mg} \mathrm{m}^{-3}$

Mo $K \alpha$ radiation, $\lambda=0.71075 \AA$

Cell parameters from 9084 reflections

$\theta=3.0-27.7^{\circ}$

$\mu=2.16 \mathrm{~mm}^{-1}$

$T=93 \mathrm{~K}$

Block, purple

$0.20 \times 0.08 \times 0.05 \mathrm{~mm}$

5757 independent reflections

4100 reflections with $F^{2}>2.0 \sigma\left(F^{2}\right)$

$R_{\text {int }}=0.080$

$\theta_{\max }=27.5^{\circ}, \theta_{\min }=3.0^{\circ}$

$h=-11 \rightarrow 11$

$k=-15 \rightarrow 15$

$l=-30 \rightarrow 30$

Secondary atom site location: difference Fourier map

Hydrogen site location: inferred from neighbouring sites

$\mathrm{H}$-atom parameters constrained

$w=1 /\left[\sigma^{2}\left(F_{\mathrm{o}}^{2}\right)+(0.062 P)^{2}+0.6421 P\right]$ where $P=\left(F_{\mathrm{o}}^{2}+2 F_{\mathrm{c}}^{2}\right) / 3$

$(\Delta / \sigma)_{\max }=0.001$

$\Delta \rho_{\max }=0.79$ e $\AA^{-3}$

$\Delta \rho_{\min }=-1.07$ e $\AA^{-3}$

Special details

Geometry. All esds (except the esd in the dihedral angle between two 1.s. planes) are estimated using the full covariance matrix. The cell esds are taken into account individually in the estimation of esds in distances, angles and torsion angles; correlations between esds in cell parameters are only used when they are defined by crystal symmetry. An approximate (isotropic) treatment of cell esds is used for estimating esds involving l.s. planes.

Refinement. Refinement was performed using all reflections. The weighted R-factor (wR) and goodness of fit (S) are based on $\mathrm{F}^{2}$. R-factor (gt) are based on $\mathrm{F}$. The threshold expression of $\mathrm{F}^{2}>2.0 \operatorname{sigma}\left(\mathrm{F}^{2}\right)$ is used only for calculating Rfactor (gt). 
Fractional atomic coordinates and isotropic or equivalent isotropic displacement parameters $\left(\AA^{2}\right)$

\begin{tabular}{|c|c|c|c|c|}
\hline & $x$ & $y$ & $z$ & $U_{\text {iso }} * / U_{\text {eq }}$ \\
\hline Br1 & $0.09534(4)$ & $0.75013(2)$ & $0.04399(2)$ & $0.03365(12)$ \\
\hline Mn1 & $0.37395(6)$ & $0.74975(3)$ & $0.03376(2)$ & $0.02720(14)$ \\
\hline $\mathrm{O} 1$ & $0.6977(3)$ & 0.77263 (19) & $0.02409(12)$ & $0.0455(6)$ \\
\hline $\mathrm{O} 2$ & $0.4042(4)$ & $0.8863(2)$ & $0.13644(11)$ & $0.0659(8)$ \\
\hline $\mathrm{O} 3$ & $0.3198(3)$ & $0.96864(19)$ & $-0.02110(11)$ & $0.0478(6)$ \\
\hline $\mathrm{O} 4$ & $-0.0202(3)$ & $0.4693(2)$ & $-0.19464(10)$ & $0.0522(7)$ \\
\hline $\mathrm{O5}$ & $0.1200(2)$ & $0.33393(18)$ & $-0.15029(8)$ & $0.0358(5)$ \\
\hline O6 & $0.2414(4)$ & $0.2443(2)$ & $0.17911(12)$ & $0.0616(9)$ \\
\hline $\mathrm{O} 7$ & $0.0767(3)$ & $0.25994(16)$ & $0.10327(10)$ & $0.0372(5)$ \\
\hline N1 & $0.3222(3)$ & 0.64858 (19) & $-0.03560(9)$ & $0.0255(5)$ \\
\hline N2 & $0.3736(3)$ & $0.59349(19)$ & $0.07024(9)$ & $0.0265(5)$ \\
\hline $\mathrm{C} 1$ & $0.5744(4)$ & $0.7601(2)$ & $0.02755(14)$ & $0.0331(7)$ \\
\hline $\mathrm{C} 2$ & $0.3926(4)$ & $0.8304(3)$ & $0.09774(14)$ & $0.0400(8)$ \\
\hline $\mathrm{C} 3$ & $0.3401(4)$ & $0.8825(3)$ & -0.00119 & $0.0342(7)$ \\
\hline $\mathrm{C} 4$ & $0.3949(4)$ & $0.7704(3)$ & $-0.10815(15)$ & $0.0380(8)$ \\
\hline H1 & 0.459451 & 0.809647 & -0.080705 & $0.046^{*}$ \\
\hline $\mathrm{C} 5$ & $0.3815(4)$ & $0.8050(3)$ & $-0.16335(15)$ & $0.0468(9)$ \\
\hline $\mathrm{H} 2$ & 0.437453 & 0.867719 & -0.173866 & $0.056^{*}$ \\
\hline C6 & $0.2868(5)$ & $0.7494(3)$ & $-0.20404(16)$ & $0.0489(10)$ \\
\hline H3 & 0.273651 & 0.777152 & -0.241603 & $0.059 *$ \\
\hline $\mathrm{C} 7$ & $0.2124(4)$ & $0.6553(3)$ & $-0.19061(13)$ & $0.0417(8)$ \\
\hline $\mathrm{H} 4$ & 0.151309 & 0.616168 & -0.219148 & $0.050 *$ \\
\hline $\mathrm{C} 8$ & 0.2262 & $0.6154(3)$ & $-0.13391(12)$ & $0.0308(7)$ \\
\hline C9 & $0.3148(3)$ & $0.6779(3)$ & $-0.09197(12)$ & $0.0301(6)$ \\
\hline $\mathrm{C} 10$ & $0.1627(3)$ & $0.5137(2)$ & $-0.11728(11)$ & $0.0283(6)$ \\
\hline C11 & $0.1841(3)$ & $0.4822(2)$ & -0.06195 (11) & $0.0273(6)$ \\
\hline H5 & 0.146315 & 0.412652 & -0.050550 & $0.033 *$ \\
\hline C12 & $0.2616(3)$ & $0.5524(2)$ & $-0.02203(12)$ & $0.0251(6)$ \\
\hline C13 & $0.2867(3)$ & $0.5228(2)$ & $0.03829(11)$ & $0.0253(6)$ \\
\hline C14 & $0.2265(3)$ & $0.4258(2)$ & $0.06018(12)$ & $0.0284(6)$ \\
\hline H6 & 0.161956 & 0.379064 & 0.036264 & $0.034 *$ \\
\hline $\mathrm{C} 15$ & $0.2605(3)$ & $0.3983(2)$ & $0.11581(12)$ & $0.0276(6)$ \\
\hline $\mathrm{C} 16$ & $0.3641(3)$ & $0.4668(2)$ & $0.15020(12)$ & $0.0290(6)$ \\
\hline $\mathrm{C} 17$ & $0.4186(3)$ & $0.5636(2)$ & $0.12506(11)$ & $0.0271(6)$ \\
\hline $\mathrm{C} 18$ & $0.4134(4)$ & $0.4435(3)$ & $0.20761(12)$ & $0.0348(7)$ \\
\hline H7 & 0.373534 & 0.381128 & 0.225729 & $0.042 *$ \\
\hline C19 & $0.5173(4)$ & $0.5101(3)$ & $0.23671(13)$ & $0.0392(8)$ \\
\hline H8 & 0.550979 & 0.492906 & 0.274893 & $0.047 *$ \\
\hline $\mathrm{C} 20$ & $0.5755(4)$ & $0.6038(3)$ & $0.21124(13)$ & $0.0369(8)$ \\
\hline H9 & 0.648519 & 0.649313 & 0.232167 & $0.044 *$ \\
\hline $\mathrm{C} 21$ & $0.5280(3)$ & $0.6298(3)$ & $0.15676(12)$ & $0.0322(7)$ \\
\hline H10 & 0.568787 & 0.693090 & 0.139770 & $0.039 *$ \\
\hline $\mathrm{C} 22$ & $0.0748(3)$ & $0.4380(3)$ & $-0.15902(12)$ & $0.0342(7)$ \\
\hline $\mathrm{C} 23$ & $0.0446(5)$ & 0.2485 & $-0.18631(15)$ & $0.0476(10)$ \\
\hline H11 & 0.043775 & 0.269435 & -0.226591 & $0.057^{*}$ \\
\hline
\end{tabular}




\begin{tabular}{lllll} 
H12 & -0.061144 & 0.238971 & -0.177556 & $0.057^{*}$ \\
C24 & $0.1315(5)$ & $0.1432(3)$ & $-0.17463(17)$ & $0.0592(11)$ \\
H13 & 0.081839 & 0.082438 & -0.196838 & $0.071^{*}$ \\
H14 & 0.135231 & 0.125364 & -0.134315 & $0.071^{*}$ \\
H15 & 0.234523 & 0.152836 & -0.185052 & $0.071^{*}$ \\
C25 & $0.1941(4)$ & $0.2929(3)$ & $0.13743(13)$ & $0.0324(7)$ \\
C26 & $0.0195(4)$ & $0.1492(3)$ & $0.11252(15)$ & $0.0412(8)$ \\
H16 & -0.088192 & 0.144696 & 0.097417 & $0.049 *$ \\
H17 & 0.026769 & 0.133367 & 0.153545 & $0.049 *$ \\
C27 & $0.1078(5)$ & $0.0658(3)$ & $0.0841(2)$ & $0.0663(12)$ \\
H18 & 0.059641 & -0.007362 & 0.085839 & $0.080^{*}$ \\
H19 & 0.210688 & 0.062260 & 0.103066 & $0.080^{*}$ \\
H20 & 0.111507 & 0.087199 & 0.044501 & $0.080^{*}$ \\
\hline
\end{tabular}

Atomic displacement parameters $\left(\AA^{2}\right)$

\begin{tabular}{|c|c|c|c|c|c|c|}
\hline & $U^{11}$ & $U^{22}$ & $U^{33}$ & $U^{12}$ & $U^{13}$ & $U^{23}$ \\
\hline $\mathrm{Br} 1$ & $0.0304(2)$ & 0.03101 (19) & $0.0392(2)$ & 0.00088 (13) & $0.00171(14)$ & $-0.00383(12)$ \\
\hline Mn1 & $0.0290(3)$ & $0.0223(2)$ & $0.0293(2)$ & -0.00015 & $-0.0018(2)$ & $-0.00277(17)$ \\
\hline O1 & $0.0346(15)$ & $0.0311(13)$ & $0.0702(17)$ & $0.0026(10)$ & $0.0027(13)$ & $0.0027(11)$ \\
\hline $\mathrm{O} 2$ & $0.085(2)$ & $0.0514(16)$ & $0.0556(16)$ & $0.0189(15)$ & $-0.0224(15)$ & $-0.0289(13)$ \\
\hline $\mathrm{O} 3$ & $0.0572(16)$ & $0.0252(12)$ & $0.0606(15)$ & $0.0045(11)$ & $0.0037(13)$ & $0.0056(11)$ \\
\hline $\mathrm{O} 4$ & $0.0465(15)$ & $0.0670(18)$ & $0.0383(12)$ & $0.0050(13)$ & $-0.0198(11)$ & $-0.0010(12)$ \\
\hline $\mathrm{O} 5$ & $0.0377(12)$ & $0.0377(12)$ & $0.0302(11)$ & $-0.0082(10)$ & $-0.0056(9)$ & $-0.0087(9)$ \\
\hline O6 & $0.070(2)$ & $0.0594(18)$ & $0.0497(16)$ & $-0.0249(14)$ & $-0.0218(15)$ & $0.0294(12)$ \\
\hline $\mathrm{O} 7$ & $0.0397(14)$ & $0.0282(12)$ & $0.0418(13)$ & $-0.0056(10)$ & $-0.0056(11)$ & $0.0058(9)$ \\
\hline N1 & $0.0261(13)$ & $0.0241(12)$ & $0.0259(12)$ & $0.0008(10)$ & $-0.0002(10)$ & $-0.0010(9)$ \\
\hline N2 & $0.0270(13)$ & $0.0259(12)$ & $0.0257(12)$ & $0.0024(10)$ & $-0.0014(10)$ & $-0.0011(10)$ \\
\hline $\mathrm{C} 1$ & $0.045(2)$ & $0.0181(14)$ & $0.0350(16)$ & $-0.0033(13)$ & $-0.0036(15)$ & $0.0017(12)$ \\
\hline $\mathrm{C} 2$ & 0.0393 (19) & $0.0360(18)$ & $0.0423(18)$ & $0.0075(15)$ & $-0.0079(15)$ & $-0.0064(15)$ \\
\hline $\mathrm{C} 3$ & $0.0329(17)$ & $0.0296(16)$ & $0.0394(17)$ & -0.0005 & 0.0002 (14) & $-0.0057(13)$ \\
\hline $\mathrm{C} 4$ & $0.042(2)$ & $0.0348(18)$ & $0.0369(17)$ & $0.0002(14)$ & $0.0045(15)$ & $0.0036(13)$ \\
\hline $\mathrm{C} 5$ & $0.055(2)$ & $0.043(2)$ & $0.044(2)$ & $-0.0002(18)$ & $0.0138(18)$ & $0.0119(16)$ \\
\hline C6 & $0.061(3)$ & $0.051(2)$ & $0.0350(18)$ & $0.0026(19)$ & $0.0100(18)$ & $0.0139(16)$ \\
\hline $\mathrm{C} 7$ & $0.047(2)$ & $0.050(2)$ & $0.0285(16)$ & $0.0056(17)$ & $0.0015(15)$ & 0.0047 (14) \\
\hline $\mathrm{C} 8$ & $0.0326(17)$ & $0.0323(16)$ & $0.0268(14)$ & $0.0072(13)$ & $0.0000(13)$ & $0.0004(12)$ \\
\hline C9 & $0.0298(16)$ & $0.0298(15)$ & $0.0301(15)$ & $0.0060(13)$ & $0.0009(12)$ & 0.0057 (12) \\
\hline $\mathrm{C} 10$ & $0.0251(15)$ & $0.0330(16)$ & $0.0260(14)$ & $0.0062(13)$ & $-0.0011(12)$ & $0.0001(12)$ \\
\hline $\mathrm{C} 11$ & $0.0275(15)$ & $0.0263(14)$ & $0.0272(14)$ & $0.0022(12)$ & $-0.0022(12)$ & $0.0001(12)$ \\
\hline $\mathrm{C} 12$ & $0.0259(15)$ & $0.0248(14)$ & $0.0240(13)$ & $0.0029(12)$ & $-0.0002(11)$ & $0.0022(11)$ \\
\hline $\mathrm{C} 13$ & $0.0272(15)$ & $0.0216(14)$ & $0.0260(13)$ & 0.0005 (12) & $-0.0022(11)$ & $-0.0018(11)$ \\
\hline C14 & $0.0311(16)$ & 0.0259 (14) & $0.0267(14)$ & 0.0003 (13) & $-0.0037(12)$ & $-0.0010(11)$ \\
\hline $\mathrm{C} 15$ & $0.0271(16)$ & $0.0260(14)$ & $0.0291(14)$ & $0.0018(12)$ & 0.0002 (12) & 0.0007 (11) \\
\hline $\mathrm{C} 16$ & $0.0298(16)$ & $0.0322(16)$ & 0.0239 (13) & 0.0048 (13) & $-0.0027(12)$ & $-0.0008(12)$ \\
\hline $\mathrm{C} 17$ & $0.0296(16)$ & $0.0289(15)$ & $0.0218(13)$ & 0.0018 (13) & $-0.0021(12)$ & $-0.0022(11)$ \\
\hline $\mathrm{C} 18$ & 0.0425 (19) & $0.0378(17)$ & $0.0236(14)$ & $0.0040(15)$ & $0.0002(13)$ & $0.0015(12)$ \\
\hline C19 & $0.046(2)$ & $0.045(2)$ & $0.0246(14)$ & 0.0085 (17) & $-0.0044(14)$ & $-0.0025(14)$ \\
\hline $\mathrm{C} 20$ & $0.0384(18)$ & $0.0401(18)$ & $0.0300(16)$ & $0.0040(15)$ & $-0.0079(14)$ & $-0.0103(13)$ \\
\hline
\end{tabular}




\begin{tabular}{lllllll}
\hline & & & & & \\
$\mathrm{C} 21$ & $0.0315(17)$ & $0.0310(16)$ & $0.0327(15)$ & $0.0005(13)$ & $-0.0025(13)$ & $-0.0033(13)$ \\
$\mathrm{C} 22$ & $0.0305(17)$ & $0.0468(19)$ & $0.0246(14)$ & $0.0010(15)$ & $-0.0010(13)$ & $-0.0032(13)$ \\
$\mathrm{C} 23$ & $0.051(2)$ & $0.057(2)$ & $0.0325(17)$ & $-0.0223(18)$ & $-0.0034(17)$ & $-0.0177(15)$ \\
$\mathrm{C} 24$ & $0.075(3)$ & $0.045(2)$ & $0.056(2)$ & $-0.016(2)$ & $0.000(2)$ & $-0.0206(18)$ \\
$\mathrm{C} 25$ & $0.0337(18)$ & $0.0309(15)$ & $0.0318(16)$ & $-0.0003(14)$ & $-0.0003(13)$ & $0.0052(13)$ \\
$\mathrm{C} 26$ & $0.045(2)$ & $0.0267(16)$ & $0.051(2)$ & $-0.0054(15)$ & $0.0005(16)$ & $0.0047(14)$ \\
$\mathrm{C} 27$ & $0.080(3)$ & $0.0310(19)$ & $0.091(3)$ & $0.003(2)$ & $0.023(3)$ & $-0.001(2)$ \\
\hline
\end{tabular}

Geometric parameters $\left(\stackrel{A}{\circ}{ }^{o}\right)$

\begin{tabular}{|c|c|c|c|}
\hline $\mathrm{Br} 1-\mathrm{Mn} 1$ & $2.5146(6)$ & $\mathrm{C} 10-\mathrm{C} 22$ & $1.506(4)$ \\
\hline $\mathrm{Mn} 1-\mathrm{C} 2$ & $1.798(3)$ & $\mathrm{C} 11-\mathrm{C} 12$ & $1.398(4)$ \\
\hline $\mathrm{Mn} 1-\mathrm{C} 1$ & $1.809(4)$ & C11-H5 & 0.9500 \\
\hline $\mathrm{Mn} 1-\mathrm{C} 3$ & $1.809(3)$ & $\mathrm{C} 12-\mathrm{C} 13$ & $1.473(4)$ \\
\hline $\mathrm{Mn} 1-\mathrm{N} 1$ & $2.063(2)$ & $\mathrm{C} 13-\mathrm{C} 14$ & $1.404(4)$ \\
\hline $\mathrm{Mn} 1-\mathrm{N} 2$ & $2.068(2)$ & $\mathrm{C} 14-\mathrm{C} 15$ & $1.368(4)$ \\
\hline $\mathrm{O} 1-\mathrm{C} 1$ & $1.118(4)$ & $\mathrm{C} 14-\mathrm{H} 6$ & 0.9500 \\
\hline $\mathrm{O} 2-\mathrm{C} 2$ & $1.135(4)$ & $\mathrm{C} 15-\mathrm{C} 16$ & $1.429(4)$ \\
\hline $\mathrm{O} 3-\mathrm{C} 3$ & $1.144(4)$ & $\mathrm{C} 15-\mathrm{C} 25$ & $1.508(4)$ \\
\hline $\mathrm{O} 4-\mathrm{C} 22$ & $1.195(4)$ & $\mathrm{C} 16-\mathrm{C} 17$ & $1.416(4)$ \\
\hline $\mathrm{O} 5-\mathrm{C} 22$ & $1.323(4)$ & $\mathrm{C} 16-\mathrm{C} 18$ & $1.419(4)$ \\
\hline $\mathrm{O} 5-\mathrm{C} 23$ & $1.457(4)$ & $\mathrm{C} 17-\mathrm{C} 21$ & $1.414(4)$ \\
\hline $\mathrm{O} 6-\mathrm{C} 25$ & $1.190(4)$ & $\mathrm{C} 18-\mathrm{C} 19$ & $1.358(4)$ \\
\hline $\mathrm{O} 7-\mathrm{C} 25$ & $1.318(4)$ & $\mathrm{C} 18-\mathrm{H} 7$ & 0.9500 \\
\hline $\mathrm{O} 7-\mathrm{C} 26$ & $1.449(4)$ & $\mathrm{C} 19-\mathrm{C} 20$ & $1.402(5)$ \\
\hline $\mathrm{N} 1-\mathrm{C} 12$ & $1.328(4)$ & C19-H8 & 0.9500 \\
\hline N1-C9 & $1.382(4)$ & $\mathrm{C} 20-\mathrm{C} 21$ & $1.358(4)$ \\
\hline $\mathrm{N} 2-\mathrm{C} 13$ & $1.333(3)$ & $\mathrm{C} 20-\mathrm{H} 9$ & 0.9500 \\
\hline $\mathrm{N} 2-\mathrm{C} 17$ & $1.373(3)$ & $\mathrm{C} 21-\mathrm{H} 10$ & 0.9500 \\
\hline $\mathrm{C} 4-\mathrm{C} 5$ & $1.371(5)$ & $\mathrm{C} 23-\mathrm{C} 24$ & $1.493(5)$ \\
\hline $\mathrm{C} 4-\mathrm{C} 9$ & $1.394(4)$ & $\mathrm{C} 23-\mathrm{H} 11$ & 0.9900 \\
\hline $\mathrm{C} 4-\mathrm{H} 1$ & 0.9500 & $\mathrm{C} 23-\mathrm{H} 12$ & 0.9900 \\
\hline $\mathrm{C} 5-\mathrm{C} 6$ & $1.389(6)$ & $\mathrm{C} 24-\mathrm{H} 13$ & 0.9800 \\
\hline $\mathrm{C} 5-\mathrm{H} 2$ & 0.9500 & $\mathrm{C} 24-\mathrm{H} 14$ & 0.9800 \\
\hline $\mathrm{C} 6-\mathrm{C} 7$ & $1.363(5)$ & $\mathrm{C} 24-\mathrm{H} 15$ & 0.9800 \\
\hline $\mathrm{C} 6-\mathrm{H} 3$ & 0.9500 & $\mathrm{C} 26-\mathrm{C} 27$ & $1.478(5)$ \\
\hline $\mathrm{C} 7-\mathrm{C} 8$ & $1.425(4)$ & $\mathrm{C} 26-\mathrm{H} 16$ & 0.9900 \\
\hline $\mathrm{C} 7-\mathrm{H} 4$ & 0.9500 & $\mathrm{C} 26-\mathrm{H} 17$ & 0.9900 \\
\hline $\mathrm{C} 8-\mathrm{C} 10$ & $1.418(4)$ & $\mathrm{C} 27-\mathrm{H} 18$ & 0.9800 \\
\hline $\mathrm{C} 8-\mathrm{C} 9$ & $1.422(4)$ & C27-H19 & 0.9800 \\
\hline $\mathrm{C} 10-\mathrm{C} 11$ & $1.364(4)$ & $\mathrm{C} 27-\mathrm{H} 20$ & 0.9800 \\
\hline $\mathrm{C} 2-\mathrm{Mn} 1-\mathrm{C} 1$ & $91.40(15)$ & $\mathrm{C} 14-\mathrm{C} 13-\mathrm{C} 12$ & $122.4(2)$ \\
\hline $\mathrm{C} 2-\mathrm{Mn} 1-\mathrm{C} 3$ & $84.91(15)$ & $\mathrm{C} 15-\mathrm{C} 14-\mathrm{C} 13$ & $120.3(3)$ \\
\hline $\mathrm{C} 1-\mathrm{Mn} 1-\mathrm{C} 3$ & 91.23 (14) & $\mathrm{C} 15-\mathrm{C} 14-\mathrm{H} 6$ & 119.9 \\
\hline $\mathrm{C} 2-\mathrm{Mn} 1-\mathrm{N} 1$ & $171.30(13)$ & $\mathrm{C} 13-\mathrm{C} 14-\mathrm{H} 6$ & 119.9 \\
\hline $\mathrm{C} 1-\mathrm{Mn} 1-\mathrm{N} 1$ & $96.74(12)$ & $\mathrm{C} 14-\mathrm{C} 15-\mathrm{C} 16$ & $118.8(3)$ \\
\hline $\mathrm{C} 3-\mathrm{Mn} 1-\mathrm{N} 1$ & $97.94(11)$ & $\mathrm{C} 14-\mathrm{C} 15-\mathrm{C} 25$ & 118.5 \\
\hline
\end{tabular}




\begin{tabular}{|c|c|c|c|}
\hline $\mathrm{C} 2-\mathrm{Mn} 1-\mathrm{N} 2$ & $97.89(12)$ & $\mathrm{C} 16-\mathrm{C} 15-\mathrm{C} 25$ & $122.6(3)$ \\
\hline $\mathrm{C} 1-\mathrm{Mn} 1-\mathrm{N} 2$ & 98.04 (11) & $\mathrm{C} 17-\mathrm{C} 16-\mathrm{C} 18$ & $118.9(3)$ \\
\hline $\mathrm{C} 3-\mathrm{Mn} 1-\mathrm{N} 2$ & $170.22(12)$ & $\mathrm{C} 17-\mathrm{C} 16-\mathrm{C} 15$ & $117.3(2)$ \\
\hline $\mathrm{N} 1-\mathrm{Mn} 1-\mathrm{N} 2$ & $77.97(9)$ & $\mathrm{C} 18-\mathrm{C} 16-\mathrm{C} 15$ & $123.8(3)$ \\
\hline $\mathrm{C} 2-\mathrm{Mn} 1-\mathrm{Br} 1$ & $85.68(11)$ & $\mathrm{N} 2-\mathrm{C} 17-\mathrm{C} 21$ & $118.6(3)$ \\
\hline $\mathrm{C} 1-\mathrm{Mn} 1-\mathrm{Br} 1$ & $175.86(9)$ & $\mathrm{N} 2-\mathrm{C} 17-\mathrm{C} 16$ & $122.5(3)$ \\
\hline $\mathrm{C} 3-\mathrm{Mn} 1-\mathrm{Br} 1$ & $85.59(10)$ & $\mathrm{C} 21-\mathrm{C} 17-\mathrm{C} 16$ & $118.9(3)$ \\
\hline $\mathrm{N} 1-\mathrm{Mn} 1-\mathrm{Br} 1$ & $86.34(7)$ & $\mathrm{C} 19-\mathrm{C} 18-\mathrm{C} 16$ & $120.2(3)$ \\
\hline $\mathrm{N} 2-\mathrm{Mn} 1-\mathrm{Br} 1$ & $85.29(7)$ & $\mathrm{C} 19-\mathrm{C} 18-\mathrm{H} 7$ & 119.9 \\
\hline $\mathrm{C} 22-\mathrm{O} 5-\mathrm{C} 23$ & $117.3(3)$ & $\mathrm{C} 16-\mathrm{C} 18-\mathrm{H} 7$ & 119.9 \\
\hline $\mathrm{C} 25-\mathrm{O} 7-\mathrm{C} 26$ & $116.8(2)$ & $\mathrm{C} 18-\mathrm{C} 19-\mathrm{C} 20$ & $121.0(3)$ \\
\hline $\mathrm{C} 12-\mathrm{N} 1-\mathrm{C} 9$ & $118.5(2)$ & $\mathrm{C} 18-\mathrm{C} 19-\mathrm{H} 8$ & 119.5 \\
\hline $\mathrm{C} 12-\mathrm{N} 1-\mathrm{Mn} 1$ & $112.39(18)$ & $\mathrm{C} 20-\mathrm{C} 19-\mathrm{H} 8$ & 119.5 \\
\hline C9-N1-Mn1 & $127.65(19)$ & $\mathrm{C} 21-\mathrm{C} 20-\mathrm{C} 19$ & $120.2(3)$ \\
\hline $\mathrm{C} 13-\mathrm{N} 2-\mathrm{C} 17$ & $118.1(2)$ & $\mathrm{C} 21-\mathrm{C} 20-\mathrm{H} 9$ & 119.9 \\
\hline $\mathrm{C} 13-\mathrm{N} 2-\mathrm{Mn} 1$ & $111.33(18)$ & $\mathrm{C} 19-\mathrm{C} 20-\mathrm{H} 9$ & 119.9 \\
\hline $\mathrm{C} 17-\mathrm{N} 2-\mathrm{Mn} 1$ & $128.58(19)$ & $\mathrm{C} 20-\mathrm{C} 21-\mathrm{C} 17$ & $120.7(3)$ \\
\hline $\mathrm{O} 1-\mathrm{C} 1-\mathrm{Mn} 1$ & $176.2(3)$ & $\mathrm{C} 20-\mathrm{C} 21-\mathrm{H} 10$ & 119.6 \\
\hline $\mathrm{O} 2-\mathrm{C} 2-\mathrm{Mn} 1$ & $176.4(3)$ & $\mathrm{C} 17-\mathrm{C} 21-\mathrm{H} 10$ & 119.6 \\
\hline $\mathrm{O} 3-\mathrm{C} 3-\mathrm{Mn} 1$ & $177.1(3)$ & $\mathrm{O} 4-\mathrm{C} 22-\mathrm{O} 5$ & $126.2(3)$ \\
\hline $\mathrm{C} 5-\mathrm{C} 4-\mathrm{C} 9$ & $120.6(3)$ & $\mathrm{O} 4-\mathrm{C} 22-\mathrm{C} 10$ & $124.1(3)$ \\
\hline $\mathrm{C} 5-\mathrm{C} 4-\mathrm{H} 1$ & 119.7 & $\mathrm{O} 5-\mathrm{C} 22-\mathrm{C} 10$ & $109.7(2)$ \\
\hline $\mathrm{C} 9-\mathrm{C} 4-\mathrm{H} 1$ & 119.7 & $\mathrm{O} 5-\mathrm{C} 23-\mathrm{C} 24$ & $106.7(3)$ \\
\hline $\mathrm{C} 4-\mathrm{C} 5-\mathrm{C} 6$ & $120.6(3)$ & $\mathrm{O} 5-\mathrm{C} 23-\mathrm{H} 11$ & 110.4 \\
\hline $\mathrm{C} 4-\mathrm{C} 5-\mathrm{H} 2$ & 119.7 & $\mathrm{C} 24-\mathrm{C} 23-\mathrm{H} 11$ & 110.4 \\
\hline $\mathrm{C} 6-\mathrm{C} 5-\mathrm{H} 2$ & 119.7 & $\mathrm{O} 5-\mathrm{C} 23-\mathrm{H} 12$ & 110.4 \\
\hline $\mathrm{C} 7-\mathrm{C} 6-\mathrm{C} 5$ & $120.7(3)$ & $\mathrm{C} 24-\mathrm{C} 23-\mathrm{H} 12$ & 110.4 \\
\hline $\mathrm{C} 7-\mathrm{C} 6-\mathrm{H} 3$ & 119.7 & $\mathrm{H} 11-\mathrm{C} 23-\mathrm{H} 12$ & 108.6 \\
\hline $\mathrm{C} 5-\mathrm{C} 6-\mathrm{H} 3$ & 119.7 & $\mathrm{C} 23-\mathrm{C} 24-\mathrm{H} 13$ & 109.5 \\
\hline $\mathrm{C} 6-\mathrm{C} 7-\mathrm{C} 8$ & $120.2(3)$ & $\mathrm{C} 23-\mathrm{C} 24-\mathrm{H} 14$ & 109.5 \\
\hline $\mathrm{C} 6-\mathrm{C} 7-\mathrm{H} 4$ & 119.9 & $\mathrm{H} 13-\mathrm{C} 24-\mathrm{H} 14$ & 109.5 \\
\hline $\mathrm{C} 8-\mathrm{C} 7-\mathrm{H} 4$ & 119.9 & $\mathrm{C} 23-\mathrm{C} 24-\mathrm{H} 15$ & 109.5 \\
\hline $\mathrm{C} 10-\mathrm{C} 8-\mathrm{C} 9$ & $117.9(3)$ & $\mathrm{H} 13-\mathrm{C} 24-\mathrm{H} 15$ & 109.5 \\
\hline $\mathrm{C} 10-\mathrm{C} 8-\mathrm{C} 7$ & $123.7(3)$ & $\mathrm{H} 14-\mathrm{C} 24-\mathrm{H} 15$ & 109.5 \\
\hline $\mathrm{C} 9-\mathrm{C} 8-\mathrm{C} 7$ & $118.4(3)$ & $\mathrm{O} 6-\mathrm{C} 25-\mathrm{O} 7$ & $123.9(3)$ \\
\hline $\mathrm{N} 1-\mathrm{C} 9-\mathrm{C} 4$ & $119.7(3)$ & $\mathrm{O} 6-\mathrm{C} 25-\mathrm{C} 15$ & $125.3(3)$ \\
\hline $\mathrm{N} 1-\mathrm{C} 9-\mathrm{C} 8$ & $121.0(3)$ & $\mathrm{O} 7-\mathrm{C} 25-\mathrm{C} 15$ & $110.8(2)$ \\
\hline $\mathrm{C} 4-\mathrm{C} 9-\mathrm{C} 8$ & $119.3(3)$ & $\mathrm{O} 7-\mathrm{C} 26-\mathrm{C} 27$ & $110.0(3)$ \\
\hline $\mathrm{C} 11-\mathrm{C} 10-\mathrm{C} 8$ & $119.2(3)$ & $\mathrm{O} 7-\mathrm{C} 26-\mathrm{H} 16$ & 109.7 \\
\hline $\mathrm{C} 11-\mathrm{C} 10-\mathrm{C} 22$ & $118.8(3)$ & $\mathrm{C} 27-\mathrm{C} 26-\mathrm{H} 16$ & 109.7 \\
\hline $\mathrm{C} 8-\mathrm{C} 10-\mathrm{C} 22$ & $122.1(3)$ & $\mathrm{O} 7-\mathrm{C} 26-\mathrm{H} 17$ & 109.7 \\
\hline $\mathrm{C} 10-\mathrm{C} 11-\mathrm{C} 12$ & $119.9(3)$ & $\mathrm{C} 27-\mathrm{C} 26-\mathrm{H} 17$ & 109.7 \\
\hline $\mathrm{C} 10-\mathrm{C} 11-\mathrm{H} 5$ & 120.0 & $\mathrm{H} 16-\mathrm{C} 26-\mathrm{H} 17$ & 108.2 \\
\hline $\mathrm{C} 12-\mathrm{C} 11-\mathrm{H} 5$ & 120.0 & $\mathrm{C} 26-\mathrm{C} 27-\mathrm{H} 18$ & 109.5 \\
\hline $\mathrm{N} 1-\mathrm{C} 12-\mathrm{C} 11$ & $122.9(3)$ & $\mathrm{C} 26-\mathrm{C} 27-\mathrm{H} 19$ & 109.5 \\
\hline $\mathrm{N} 1-\mathrm{C} 12-\mathrm{C} 13$ & $114.9(2)$ & $\mathrm{H} 18-\mathrm{C} 27-\mathrm{H} 19$ & 109.5 \\
\hline $\mathrm{C} 11-\mathrm{C} 12-\mathrm{C} 13$ & $122.1(3)$ & $\mathrm{C} 26-\mathrm{C} 27-\mathrm{H} 20$ & 109.5 \\
\hline $\mathrm{N} 2-\mathrm{C} 13-\mathrm{C} 14$ & $122.5(3)$ & $\mathrm{H} 18-\mathrm{C} 27-\mathrm{H} 20$ & 109.5 \\
\hline
\end{tabular}




\begin{tabular}{|c|c|}
\hline $\mathrm{N} 2-\mathrm{C} 13-\mathrm{C} 12$ & $115.1(2)$ \\
\hline $\mathrm{C} 9-\mathrm{C} 4-\mathrm{C} 5-\mathrm{C} 6$ & $0.6(6)$ \\
\hline $\mathrm{C} 4-\mathrm{C} 5-\mathrm{C} 6-\mathrm{C} 7$ & $-4.0(6)$ \\
\hline $\mathrm{C} 5-\mathrm{C} 6-\mathrm{C} 7-\mathrm{C} 8$ & $2.6(6)$ \\
\hline $\mathrm{C} 6-\mathrm{C} 7-\mathrm{C} 8-\mathrm{C} 10$ & $-174.7(3)$ \\
\hline $\mathrm{C} 6-\mathrm{C} 7-\mathrm{C} 8-\mathrm{C} 9$ & $2.0(5)$ \\
\hline $\mathrm{C} 12-\mathrm{N} 1-\mathrm{C} 9-\mathrm{C} 4$ & $-171.2(3)$ \\
\hline $\mathrm{Mn} 1-\mathrm{N} 1-\mathrm{C} 9-\mathrm{C} 4$ & $23.8(4)$ \\
\hline $\mathrm{C} 12-\mathrm{N} 1-\mathrm{C} 9-\mathrm{C} 8$ & $8.9(4)$ \\
\hline $\mathrm{Mn} 1-\mathrm{N} 1-\mathrm{C} 9-\mathrm{C} 8$ & $-156.1(2)$ \\
\hline $\mathrm{C} 5-\mathrm{C} 4-\mathrm{C} 9-\mathrm{N} 1$ & $-175.8(3)$ \\
\hline $\mathrm{C} 5-\mathrm{C} 4-\mathrm{C} 9-\mathrm{C} 8$ & $4.1(5)$ \\
\hline $\mathrm{C} 10-\mathrm{C} 8-\mathrm{C} 9-\mathrm{N} 1$ & $-8.5(4)$ \\
\hline $\mathrm{C} 7-\mathrm{C} 8-\mathrm{C} 9-\mathrm{N} 1$ & $174.6(3)$ \\
\hline $\mathrm{C} 10-\mathrm{C} 8-\mathrm{C} 9-\mathrm{C} 4$ & $171.6(3)$ \\
\hline $\mathrm{C} 7-\mathrm{C} 8-\mathrm{C} 9-\mathrm{C} 4$ & $-5.3(4)$ \\
\hline $\mathrm{C} 9-\mathrm{C} 8-\mathrm{C} 10-\mathrm{C} 11$ & $2.4(4)$ \\
\hline $\mathrm{C} 7-\mathrm{C} 8-\mathrm{C} 10-\mathrm{C} 11$ & $179.1(3)$ \\
\hline $\mathrm{C} 9-\mathrm{C} 8-\mathrm{C} 10-\mathrm{C} 22$ & $-176.4(3)$ \\
\hline $\mathrm{C} 7-\mathrm{C} 8-\mathrm{C} 10-\mathrm{C} 22$ & $0.3(5)$ \\
\hline $\mathrm{C} 8-\mathrm{C} 10-\mathrm{C} 11-\mathrm{C} 12$ & $3.0(4)$ \\
\hline $\mathrm{C} 22-\mathrm{C} 10-\mathrm{C} 11-\mathrm{C} 12$ & $-178.2(3)$ \\
\hline $\mathrm{C} 9-\mathrm{N} 1-\mathrm{C} 12-\mathrm{C} 11$ & $-3.3(4)$ \\
\hline $\mathrm{Mn} 1-\mathrm{N} 1-\mathrm{C} 12-\mathrm{C} 11$ & $163.9(2)$ \\
\hline $\mathrm{C} 9-\mathrm{N} 1-\mathrm{C} 12-\mathrm{C} 13$ & $174.6(2)$ \\
\hline $\mathrm{Mn} 1-\mathrm{N} 1-\mathrm{C} 12-\mathrm{C} 13$ & $-18.2(3)$ \\
\hline $\mathrm{C} 10-\mathrm{C} 11-\mathrm{C} 12-\mathrm{N} 1$ & $-2.7(4)$ \\
\hline $\mathrm{C} 10-\mathrm{C} 11-\mathrm{C} 12-\mathrm{C} 13$ & $179.5(3)$ \\
\hline $\mathrm{C} 17-\mathrm{N} 2-\mathrm{C} 13-\mathrm{C} 14$ & $7.2(4)$ \\
\hline $\mathrm{Mn} 1-\mathrm{N} 2-\mathrm{C} 13-\mathrm{C} 14$ & $-158.3(2)$ \\
\hline $\mathrm{C} 17-\mathrm{N} 2-\mathrm{C} 13-\mathrm{C} 12$ & $-171.3(2)$ \\
\hline $\mathrm{Mn} 1-\mathrm{N} 2-\mathrm{C} 13-\mathrm{C} 12$ & $23.2(3)$ \\
\hline $\mathrm{N} 1-\mathrm{C} 12-\mathrm{C} 13-\mathrm{N} 2$ & $-3.5(4)$ \\
\hline $\mathrm{C} 11-\mathrm{C} 12-\mathrm{C} 13-\mathrm{N} 2$ & $174.4(3)$ \\
\hline $\mathrm{N} 1-\mathrm{C} 12-\mathrm{C} 13-\mathrm{C} 14$ & $178.0(3)$ \\
\hline $\mathrm{C} 11-\mathrm{C} 12-\mathrm{C} 13-\mathrm{C} 14$ & $-4.1(4)$ \\
\hline $\mathrm{N} 2-\mathrm{C} 13-\mathrm{C} 14-\mathrm{C} 15$ & $-2.4(4)$ \\
\hline
\end{tabular}

$\mathrm{H} 19-\mathrm{C} 27-\mathrm{H} 20$

109.5

$\mathrm{C} 12-\mathrm{C} 13-\mathrm{C} 14-\mathrm{C} 15$

$\mathrm{C} 13-\mathrm{C} 14-\mathrm{C} 15-\mathrm{C} 16$

$\mathrm{C} 13-\mathrm{C} 14-\mathrm{C} 15-\mathrm{C} 25$

$\mathrm{C} 14-\mathrm{C} 15-\mathrm{C} 16-\mathrm{C} 17$

$\mathrm{C} 25-\mathrm{C} 15-\mathrm{C} 16-\mathrm{C} 17$

$\mathrm{C} 14-\mathrm{C} 15-\mathrm{C} 16-\mathrm{C} 18$

$\mathrm{C} 25-\mathrm{C} 15-\mathrm{C} 16-\mathrm{C} 18$

$\mathrm{C} 13-\mathrm{N} 2-\mathrm{C} 17-\mathrm{C} 21$

$\mathrm{Mn} 1-\mathrm{N} 2-\mathrm{C} 17-\mathrm{C} 21$

$\mathrm{C} 13-\mathrm{N} 2-\mathrm{C} 17-\mathrm{C} 16$

$\mathrm{Mn} 1-\mathrm{N} 2-\mathrm{C} 17-\mathrm{C} 16$

$\mathrm{C} 18-\mathrm{C} 16-\mathrm{C} 17-\mathrm{N} 2$

$\mathrm{C} 15-\mathrm{C} 16-\mathrm{C} 17-\mathrm{N} 2$

$\mathrm{C} 18-\mathrm{C} 16-\mathrm{C} 17-\mathrm{C} 21$

$\mathrm{C} 15-\mathrm{C} 16-\mathrm{C} 17-\mathrm{C} 21$

$\mathrm{C} 17-\mathrm{C} 16-\mathrm{C} 18-\mathrm{C} 19$

C15-C16-C18-C19

$\mathrm{C} 16-\mathrm{C} 18-\mathrm{C} 19-\mathrm{C} 20$

$\mathrm{C} 18-\mathrm{C} 19-\mathrm{C} 20-\mathrm{C} 21$

$\mathrm{C} 19-\mathrm{C} 20-\mathrm{C} 21-\mathrm{C} 17$

$\mathrm{N} 2-\mathrm{C} 17-\mathrm{C} 21-\mathrm{C} 20$

$\mathrm{C} 16-\mathrm{C} 17-\mathrm{C} 21-\mathrm{C} 20$

$\mathrm{C} 23-\mathrm{O} 5-\mathrm{C} 22-\mathrm{O} 4$

$\mathrm{C} 23-\mathrm{O} 5-\mathrm{C} 22-\mathrm{C} 10$

$\mathrm{C} 11-\mathrm{C} 10-\mathrm{C} 22-\mathrm{O} 4$

$\mathrm{C} 8-\mathrm{C} 10-\mathrm{C} 22-\mathrm{O} 4$

$\mathrm{C} 11-\mathrm{C} 10-\mathrm{C} 22-\mathrm{O} 5$

$\mathrm{C} 8-\mathrm{C} 10-\mathrm{C} 22-\mathrm{O} 5$

$\mathrm{C} 22-\mathrm{O} 5-\mathrm{C} 23-\mathrm{C} 24$

$\mathrm{C} 26-\mathrm{O} 7-\mathrm{C} 25-\mathrm{O} 6$

$\mathrm{C} 26-\mathrm{O} 7-\mathrm{C} 25-\mathrm{C} 15$

$\mathrm{C} 14-\mathrm{C} 15-\mathrm{C} 25-\mathrm{O} 6$

$\mathrm{C} 16-\mathrm{C} 15-\mathrm{C} 25-\mathrm{O} 6$

$\mathrm{C} 14-\mathrm{C} 15-\mathrm{C} 25-\mathrm{O} 7$

$\mathrm{C} 16-\mathrm{C} 15-\mathrm{C} 25-\mathrm{O} 7$

$\mathrm{C} 25-\mathrm{O} 7-\mathrm{C} 26-\mathrm{C} 27$
$176.0(3)$

$-3.2(4)$

$-179.7(3)$

$3.7(4)$

$180.0(3)$

$-176.8(3)$

$-0.5(5)$

$170.9(3)$

$-26.4(4)$

$-6.5(4)$

$156.1(2)$

$-178.4(3)$

$1.1(4)$

$4.2(4)$

$-176.3(3)$

$-3.5(4)$

$177.0(3)$

$1.2(5)$

$0.3(5)$

$0.5(5)$

$179.7(3)$

$-2.8(4)$

$-1.8(5)$

$178.8(3)$

$135.9(3)$

$-45.3(5)$

-44.7 (4)

$134.1(3)$

$171.0(3)$

$-10.5(5)$

$168.4(3)$

$159.9(4)$

$-16.4(5)$

$-18.9(4)$

$164.7(3)$

-83.7 (4)

Hydrogen-bond geometry $\left(\AA,{ }^{\circ}\right)$

\begin{tabular}{lllll}
\hline$D-\mathrm{H} \cdots A$ & $D-\mathrm{H}$ & $\mathrm{H} \cdots A$ & $D \cdots A$ & $D-\mathrm{H} \cdots A$ \\
\hline $\mathrm{C} 7-\mathrm{H} 4 \cdots \mathrm{O} 4$ & 0.95 & 2.44 & $3.040(4)$ & 121 \\
$\mathrm{C} 11-\mathrm{H} 5 \cdots \mathrm{Br} 1^{\mathrm{i}}$ & 0.95 & 2.92 & $3.789(3)$ & 153 \\
$\mathrm{C} 14-\mathrm{H} 6 \cdots \mathrm{O} 7$ & 0.95 & 2.33 & $2.659(3)$ & 100 \\
$\mathrm{C} 18-\mathrm{H} 7 \cdots \mathrm{O} 6$ & 0.95 & 2.25 & $2.883(5)$ & 124
\end{tabular}


supporting information

\begin{tabular}{llllr}
$\mathrm{C} 19-\mathrm{H} 8 \cdots \mathrm{O} 2^{\mathrm{ii}}$ & 0.95 & 2.47 & $3.373(4)$ & 160 \\
$\mathrm{C} 20-\mathrm{H} 9 \cdots \mathrm{O}^{\mathrm{iii}}$ & 0.95 & 2.51 & $3.383(4)$ & 153 \\
\hline
\end{tabular}

Symmetry codes: (i) $-x,-y+1,-z$; (ii) $-x+1, y-1 / 2,-z+1 / 2$; (iii) $-x+1, y+1 / 2,-z+1 / 2$. 